\title{
Dynamics of slender monopoles and anti-monopoles in non-Abelian superconductor
}

\author{
Masato Arai, ${ }^{a, b}$ Filip Blaschke, ${ }^{b, c}$ Minoru Eto ${ }^{d}$ and Norisuke Sakai ${ }^{e}$ \\ ${ }^{a}$ Fukushima National College of Technology, \\ 30 Nagao, Kamiarakawa, Taira, Iwaki, Fukushima 970-8034, Japan \\ ${ }^{b}$ Institute of Experimental and Applied Physics, Czech Technical University in Prague, \\ Horská 3a/22, 12800 Prague 2, Czech Republic \\ Institute of Physics, Silesian University in Opava, \\ Bezručovo náměstí 1150/13, 74601 Opava, Czech Republic \\ ${ }^{d}$ Department of Physics, Yamagata University, \\ Kojirakawa-machi 1-4-12, Yamagata, Yamagata 990-8560, Japan \\ e Department of Physics, and Research and Education Center for Natural Sciences, Keio University, \\ Hiyoshi 4-1-1, Yokohama, Kanagawa 223-8521, Japan \\ E-mail: masato.arai@fukushima-nct.ac.jp, fblasch@post.cz, \\ meto@sci.kj.yamagata-u.ac.jp, norisuke.sakai@gmail.com
}

AbStRaCT: Low energy dynamics of magnetic monopoles and anti-monopoles in the $\mathrm{U}(2)_{\mathrm{C}}$ gauge theory is studied in the Higgs (non-Abelian superconducting) phase. The monopoles in this superconducting phase are not spherical but are of slender ellipsoid which are pierced by a vortex string. We investigate scattering of the slender monopole and anti-monopole, and find that they do not always decay into radiation, contrary to our naive intuition. They can repel, make bound states (magnetic mesons) or resonances. Analytical solutions including any number of monopoles and anti-monopoles are obtained in the first non-trivial order of rigid-body approximation. We point out that some part of solutions of slender monopole system in $1+3$ dimensions can be mapped exactly onto the sine-Gordon system in $1+1$ dimensions. This observation allows us to visualize dynamics of monopole and anti-monopole scattering easily.

KEYwords: Solitons Monopoles and Instantons, Supersymmetric gauge theory, Field Theories in Lower Dimensions

ARXIV EPRINT: 1407.2332 


\section{Contents}

1 Introduction 1

2 Model 4

3 Monopole in the Higgs phase $\quad 5$

3.1 The BPS equations 5

$\begin{array}{lll}3.2 & \text { Vortex strings } & 7\end{array}$

$\begin{array}{ll}3.3 & \text { A monopole in the Higgs phase } \\ \end{array}$

4 Rigid-body approximation $\quad 13$

$\begin{array}{lll}4.1 & \text { Formalism } & 13\end{array}$

$\begin{array}{lll}4.2 & \text { Monopole } & 18\end{array}$

5 Dynamics of slender monopoles and anti-monopoles $\quad 20$

5.1 Brief summary of the rigid body approximation 20

5.2 A dictionary: mapping onto the sine-Gordon model 21

5.3 Two different species of slender monopoles 23

$\begin{array}{ll}5.4 \text { Magnetic meson } & 25\end{array}$

5.5 Scattering of the slender monopole and anti-monopole 26

$\begin{array}{ll}5.6 & \text { Lifting the zero mode } \\ \end{array}$

6 Dyon in the Higgs phase 31

$\begin{array}{lll}7 & \text { Conclusion and discussion } & 33\end{array}$

\section{Introduction}

Topological solitons in classical and quantum field theories are widely appreciated as important objects in diverse areas of modern physics [1-4]. Among various topological solitons, magnetic monopoles are one of the most fascinating solitons in high energy physics. If a monopole exists, electric charges in the universe are quantized by the Dirac's quantization condition [5]: product of electric charge $Q_{\mathrm{e}} e$ and magnetic charge $Q_{\mathrm{m}} g$ must be proportional to integers: $Q_{\mathrm{e}} e Q_{\mathrm{m}} g \propto n$. This implies that a weak electric coupling $e$ corresponds to a strong magnetic coupling $g$. This strong-weak coupling duality is a powerful tool to understand strong coupling physics. Furthermore, monopoles are expected to play an important role to explain the confinement in QCD. It has been proposed that the QCD vacuum is in dual color superconductor where magnetic monopoles condense [6-9]. Then the color-electric fluxes from quarks and anti-quarks are squeezed to form stringy flux tubes, resulting in a linear confining potential. It is, however, very difficult to verify this 
idea, since QCD is strongly coupled at low energies because of the asymptotic freedom. It is only in the case of supersymmetric gauge theories [10] that this confinement mechanism has been demonstrated explicitly.

In contrast, QCD becomes weakly coupled at high baryon density, and enters into the color-superconducting phase where di-quarks condense [11-13]. Then magnetic monopoles should be confined and color magnetic fields form flux tubes. The flux tube in the colorflavor locked (CFL) phase at asymptotically high density is a non-Abelian vortex string (called semi-superfluid vortex string) $[14,15]$. A distinctive feature of non-Abelian vortex compared to the usual Nielsen-Olesen Abelian vortex is that the non-Abelian vortex breaks a non-Abelian global symmetry of the vacuum state and non-Abelian orientational moduli [16-19] emerge. This orientational moduli has been found to give a confining state of monopole and anti-monopole, namely magnetic meson was predicted in high density QCD [20-22].

If we tune couplings to critical values which enable us to embed the theory into a supersymmetric one, we can have Bogomolnyi-Prasad-Sommerfield (BPS) solitons [23, 24], which preserve a part of supersymmetry [25]. An analytic solution [23, 24] can be obtained for the 't Hooft-Polyakov monopole $[26,27]$ in the Coulomb phase where SU(2) gauge symmetry is broken to a $U(1)$ subgroup. Since there is no static forces between BPS monopoles, positions of BPS monopoles become parameters of the solution, which are generically called moduli. The moduli space of monopoles must be a hyper-Kähler manifold, reflecting the underlying supersymmetry with eight supercharges. The dynamics of slowly moving BPS solitons are well-approximated by a geodesic motion on the moduli space [28]. This is called the moduli approximation. Asymptotic metrics on moduli space have been known for well-separated monopoles [29]. For the particular case of scattering of two monopoles, the exact metric is known, and it is explicitly shown that slowly-moving BPS monopoles scatter with the right angle when they collide head-on [30]. Although the moduli approximation is useful, one should note that it can be applied neither for scattering of BPS solitons with high momentum nor for non-BPS systems. Among non-BPS solutions, an interesting non-BPS "bound state" of a monopole and an anti-monopole in the Coulomb phase has been rigorously established [31]. Subsequent numerical studies also revealed a vast array of monopole and anti-monopole composite states [32-34]. Although these "bound states" eventually decay due to unstable modes, they can play a significant and remarkable role in understanding the dynamics of monopole and anti-monopole system in the Coulomb phase. It has been noted that D-brane and anti-D-brane configurations play an important role in D-brane dynamics even though they are unstable [35-37].

If the non-Abelian gauge symmetry is completely broken, we are in the Higgs phase, namely the non-Abelian superconducting phase. In contrast to the Coulomb phase, monopoles in the Higgs phase have several distinctive features. Firstly, they are pierced by a vortex string. In other words, the magnetic fields coming out of the monopole are squeezed into flux tubes attached to both ends of the monopole. In the non-Abelian superconductor, there can be several species of magnetic flux tubes and a magnetic monopole can be interpreted as a junction [38] on which two different species of magnetic fluxes meet. In fact, the static BPS monopole in the Higgs phase has been found as a static 
BPS kink solution in the $1+1$-dimensional low-energy effective field theory on the vortex [39]. Secondly, shape of a monopole is not spherical. This can be seen as follows. In the non-Abelian superconductor, there are two types of topological solitons, vortex string and monopole. Thus we have two fundamental length scales: one is a transverse size $L_{\mathrm{T}}$ of the flux tube and the other is a length $L_{\mathrm{L}}$ of the monopole. Since a monopole resides on a vortex, its shape is generally not spherical depending on the ratio of the two scales. The $1+1$ dimensional effective theory description was used in refs. $[18,19,39]$ assuming

$$
L_{\mathrm{T}} \ll L_{\mathrm{L}},
$$

where monopoles are of a slender ellipsoidal shape. This approximation has been used previously [40] to obtain an effective action of 1/4 BPS non-Abelian monopole-vortex complex. Another work to obtain effective action of monopole-vortex complex appeared recently [41].

In this work, we will consider a straight vortex string where both monopoles and antimonopoles reside. Since certain interaction exists between a monopole and anti-monopole, such solutions can no longer be static. The monopoles and anti-monopoles move along the vortex string. Therefore, scatterings are all head-on collisions. In contrast to lots of studies on scattering of the BPS monopoles, there is very few studies about the dynamics of BPS monopoles and anti-monopoles, especially in the Higgs phase. This is mainly due to the inapplicability of the moduli approximation to the non-BPS monopoles and antimonopoles system, which necessitates other methods such as numerical analysis. Instead of numerical methods, we consider a systematic expansion in powers of the ratio $L_{\mathrm{T}} / L_{\mathrm{L}}$ of length scales of the model, which allows us to obtain analytic solutions. At the first order of the expansion, we obtain the rigid-body approximation, where distortion of vortex profile can be neglected during the collision. These approximate analytic solutions are very useful to understand the dynamics of monopole and anti-monopole system. As explained above, the monopole and anti-monopole dynamics here is essentially $1+1$-dimensional. This observation is crucial in this work. We will observe that a part of the dynamics can be mapped on to the integrable sine-Gordon model. In the rigid-body approximation, we can compute magnetic and electric fields and other induced fields with the aid of an exact mapping between $1+3$-dimensional field configurations of gauge theory and 1+1-dimensional field configurations of the sine-Gordon model. This allows us to visualize scattering of monopoles and anti-monopoles easily. As a result, we obtain that monopoles and anti-monopoles can repel each other, make bound states (magnetic mesons), or resonances, as in the case of dense QCD [20-22]. Another reason why there are only few studies of monopole and anti-monopole system may be possible instabilities of the system. Since the total topological charge for the monopole and anti-monopole pair vanishes, one may be tempted to conclude that they eventually annihilate into radiation. In spite of this intuition, we find an intriguing result that they can repel, make bound states or resonances. Thus, our study may open a new direction in understanding the monopole dynamics.

This paper is organized as follows. The model is introduced in section 2, where we also discuss the geometry of the vacuum manifold and of the associated moduli space. In section 3 we derive BPS equations and describe two types of static solutions, before the exact $1 / 2$ BPS vortex string solution and the approximate 1/4 BPS solution, describing a static 
monopole attached to the host vortex string. In section 4 , we rederive these solutions using a systematic expansion in powers of $L_{\mathrm{T}} / L_{\mathrm{L}}$. At the first order of the expansion we obtain the rigid-body approximation. We provide a mapping between $1+3$-dimensional dynamics onto a $1+1$-dimensional effective dynamics. In section 5 we fully utilize this mapping in order to discuss scattering of monopoles and anti-monopoles on the vortex string and also their bound states. In section 6 we study dyonic extension of monopoles by using the rigidbody approximation. We conclude and discuss future directions of our work in section 7 .

\section{Model}

Let us consider a U(2) $\mathrm{C}$ Yang-Mills-Higgs system

$$
\begin{aligned}
& \mathcal{L}=\operatorname{Tr}\left[-\frac{1}{2 g^{2}} F_{\mu \nu} F^{\mu \nu}+D_{\mu} H\left(D^{\mu} H\right)^{\dagger}+\frac{1}{g^{2}} D_{\mu} \Sigma D^{\mu} \Sigma\right]-V, \\
& V=\operatorname{Tr}\left[\frac{g^{2}}{4}\left(H H^{\dagger}-v^{2} \mathbf{1}_{2}\right)^{2}+(\Sigma H-H M)(\Sigma H-H M)^{\dagger}\right],
\end{aligned}
$$

where the field strength and the covariant derivatives are defined by

$$
\begin{aligned}
F_{\mu \nu} & =\partial_{\mu} A_{\nu}-\partial_{\nu} A_{\mu}+i\left[A_{\mu}, A_{\nu}\right], \\
D_{\mu} H & =\partial_{\mu} H+i A_{\mu} H, \\
D_{\mu} \Sigma & =\partial_{\mu} \Sigma+i\left[A_{\mu}, \Sigma\right] .
\end{aligned}
$$

The $N_{F}$ species of Higgs fields in the fundamental representation of the $\mathrm{U}(2)_{\mathrm{C}}$ gauge group is denoted by a $2 \times N_{\mathrm{F}}$ matrix $H$. We concentrate on $N_{\mathrm{F}}=2$ case in the following. Another Higgs field $\Sigma$ in the adjoint representation of the $\mathrm{U}(2)_{\mathrm{C}}$ gauge group is denoted by a real $2 \times 2$ matrix. The quartic scalar coupling is given in terms of the gauge coupling constant $g$, which allows our model to be embeded in a supersymmetric theory. The parameter $v$ giving the vacuum expectation value of the Higgs field $H$ comes from the so-called FayetIlliopoulos (FI) parameter in the supersymmetric context. We assume $v>0$ in what follows. We take the mass matrix $M$ for $H$ in the following form

$$
M=\frac{m}{2} \sigma_{3} .
$$

Global symmetry of the model depends on the mass parameter $m$. If $m=0$, the flavor symmetry is $\mathrm{SU}(2)_{\mathrm{F}}$. If $m \neq 0$, the flavor symmetry reduces to $\mathrm{U}(1)_{\mathrm{F}} \subset \mathrm{SU}(2)_{\mathrm{F}}$ generated by the third component of $\mathrm{SU}(2)_{\mathrm{F}}$.

The vacuum of the model is determined by the condition $V=0$ :

$$
H H^{\dagger}=v^{2} \mathbf{1}_{2}, \quad \Sigma=M .
$$

Fixing $\Sigma=M$, the most general solution for $H$ is given for $m \neq 0$ by

$$
H=v e^{i \alpha} e^{i \beta \sigma_{3} / 2},
$$

and for $m=0$ in terms of $U \in \mathrm{U}(2)$ as

$$
H=v U .
$$


Therefore the vacuum manifold in the $m \neq 0$ case is

$$
\frac{\frac{\mathrm{U}(1)_{\mathrm{C} 0} \times \mathrm{U}(1)_{\mathrm{C} 3}}{\mathbb{Z}_{2}} \times \mathrm{U}(1)_{\mathrm{F}}}{\mathrm{U}(1)_{\mathrm{C} 3+\mathrm{F}}} \simeq \frac{\mathrm{U}(1) \times \mathrm{U}(1)}{\mathbb{Z}_{2}} \simeq T^{2},
$$

and in the $m=0$ case is given as

$$
\frac{\mathrm{U}(2)_{\mathrm{C}} \times \mathrm{SU}(2)_{\mathrm{F}}}{\mathrm{SU}(2)_{\mathrm{C}+\mathrm{F}}} \simeq \mathrm{U}(2) .
$$

In the massive case $\mathrm{U}(1)_{\mathrm{C} 0}$ stands for the overall $\mathrm{U}(1)$ subgroup of $\mathrm{U}(2)_{\mathrm{C}}$, while $\mathrm{U}(1)_{\mathrm{C} 3}$ is a $\mathrm{U}(1)$ subgroup of $\mathrm{SU}(2)_{\mathrm{C}}$ generated by $\sigma_{3} / 2$. The $\mathrm{U}(1)_{\mathrm{C} 3+\mathrm{F}}$ is a diagonal group of simultaneous $\mathrm{U}(1)_{\mathrm{C} 3}$ and $\mathrm{U}(1)_{\mathrm{F}}$ rotations which is unbroken by the vacuum. The $\mathbb{Z}_{2}$ center of $\mathrm{U}(2)_{\mathrm{C}}$ here is needed to prevent over-counting, since in eq. (2.8) both $H=e^{i \alpha} e^{i \beta \sigma_{3} / 2}$ and $H=e^{i(\alpha+\pi)} e^{i(\beta+\pi) \sigma_{3} / 2}$ represent the same point on the vacuum manifold. In either massive or massless case, however, we can make $\mathrm{U}(2)_{\mathrm{C}}$ gauge transformations to bring the fields to the following representative value

$$
H=v \mathbf{1}_{2}, \quad \Sigma=M .
$$

Therefore all the points in the vacuum manifold are physically equivalent, and the vacuum moduli space consists of only one point. We call this vacuum the color-flavor locking (CFL) vacuum. The vacuum is in the Higgs phase, where the gauge symmetry is completely broken.

In this work, we consider $m \neq 0$ case unless stated otherwise.

\section{Monopole in the Higgs phase}

\subsection{The BPS equations}

The model (2.1) admits rich topological excitations; vortex strings and magnetic monopoles. In the Higgs vacuum, magnetic field can only exist by having an unbroken normal vacuum in a small neighborhood of the zero of the Higgs field. Hence magnetic field is squeezed into a vortex, which we call a vortex string. The vortex string is topologically stable due to a non-trivial fundamental homotopy group in the massive case

$$
\pi_{1}\left(T^{2}\right)=\mathbb{Z} \times \mathbb{Z}
$$

There are two kinds of vortex quantum numbers, corresponding to two kinds of vortex strings, which we call the N-vortex and S-vortex. A magnetic monopole is a source of the conserved magnetic fluxes which are squeezed into vortex strings in this Higgs vacuum. Therefore a stable magnetic monopole is possible only as a composite soliton in the middle of a vortex string, but cannot exist as an isolated soliton, which can also be understood from the trivial homotopy

$$
\pi_{2}\left(T^{2}\right)=0
$$


In the $\mathrm{U}(2)_{\mathrm{C}}$ Yang-Mills Higgs theory, solutions for the magnetic monopole pierced by vortex strings have been found [39, 42], which preserve a quarter of supersymmetry charges when embedded into the supersymmetric theory. To see these Bogomol'nyi-PrasadSommerfield (BPS) solutions, we rewrite the total energy $E$ of static fields (and $A_{0}=0$ ) as a sum of perfect squares (plus boundary terms) as follows

$$
\begin{aligned}
E=\int d^{3} x \operatorname{Tr}[ & \frac{1}{g^{2}}\left\{\left(F_{12}-D_{3} \Sigma-\frac{g^{2}}{2}\left(H H^{\dagger}-v^{2} \mathbf{1}_{2}\right)\right)^{2}+\left(F_{23}-D_{1} \Sigma\right)^{2}+\left(F_{31}-D_{2} \Sigma\right)^{2}\right\} \\
& +4 \bar{D} H(\bar{D} H)^{\dagger}+\left(D_{3} H+\Sigma H-H M\right)\left(D_{3} H+\Sigma H-H M\right)^{\dagger} \\
& \left.+\frac{1}{g^{2}} \epsilon_{i j k} \partial_{i}\left(\Sigma F_{j k}\right)-v^{2} F_{12}+i\left\{\partial_{1}\left(H D_{2} H^{\dagger}\right)-\partial_{2}\left(H D_{1} H^{\dagger}\right)\right\}\right] .
\end{aligned}
$$

We define

$$
\begin{array}{llll}
z=x^{1}+i x^{2}, & \bar{z}=x^{1}-i x^{2}, & \partial=\frac{\partial_{1}-i \partial_{2}}{2}, & \bar{\partial}=\frac{\partial_{1}+i \partial_{2}}{2}, \\
A=\frac{A_{1}-i A_{2}}{2}, & \bar{A}=\frac{A_{1}+i A_{2}}{2}, & D=\frac{D_{1}-i D_{2}}{2}, & \bar{D}=\frac{D_{1}+i D_{2}}{2} .
\end{array}
$$

The last term in eq. (3.3) is the total derivative term which does not contribute to the total energy. In deriving eq. (3.3), we have used the following identities

$$
\begin{aligned}
D_{i} H\left(D_{i} H\right)^{\dagger} & =2\left(D H(D H)^{\dagger}+\bar{D} H(\bar{D} H)^{\dagger}\right), \\
D H(D H)^{\dagger} & =\bar{D} H(\bar{D} H)^{\dagger}-\frac{1}{2} H H^{\dagger} F_{12}+\frac{i}{2}\left\{\partial_{1}\left(H\left(D_{2} H\right)^{\dagger}\right)-\partial_{2}\left(H\left(D_{1} H\right)^{\dagger}\right)\right\} .
\end{aligned}
$$

Other useful formulas are

$$
\begin{aligned}
{\left[D_{1}, D_{2}\right] } & =-i F_{12}, \\
\partial\left(H(D H)^{\dagger}\right)-\bar{\partial}\left(H(\bar{D} H)^{\dagger}\right) & =\frac{i}{2}\left\{\partial_{1}\left(H\left(D_{2} H\right)^{\dagger}\right)-\partial_{2}\left(H\left(D_{1} H\right)^{\dagger}\right)\right\} .
\end{aligned}
$$

The total energy $E$ is bounded by the sum of two topological charges representing the monopole energy $M_{\text {mono }}$ and the vortex energy $M_{\text {vor }}$

$$
\begin{aligned}
E & \geq M_{\text {mono }}+M_{\text {vor }}, \\
M_{\text {mono }} & =\frac{1}{g^{2}} \int d^{3} x \operatorname{Tr}\left[\epsilon_{i j k} \partial_{i}\left(\Sigma F_{j k}\right)\right], \\
M_{\text {vor }} & =-v^{2} \int d^{3} x \operatorname{Tr}\left[F_{12}\right] .
\end{aligned}
$$

This bound is saturated when the following BPS equations are satisfied

$$
\begin{aligned}
F_{12}-D_{3} \Sigma-\frac{g^{2}}{2}\left(H H^{\dagger}-v \mathbf{1}_{2}\right) & =0, \\
F_{23}-D_{1} \Sigma & =0, \\
F_{31}-D_{2} \Sigma & =0,
\end{aligned}
$$




$$
\begin{aligned}
\bar{D} H & =0, \\
D_{3} H+\Sigma H-H M & =0 .
\end{aligned}
$$

If we define a $2 \times 2$ matrix field $S$ taking values in $G L(2, \mathbb{C})$ whose elements are functions of $x^{1,2,3}$ as

$$
\bar{A}=-i S^{-1} \bar{\partial} S, \quad A_{3}-i \Sigma=-i S^{-1} \partial_{3} S,
$$

we can solve the equations (3.14)-(3.17) in terms of a holomorphic matrix $H_{0}(z)$

$$
H=v S^{-1}\left(x^{1}, x^{2}, x^{3}\right) H_{0}(z) e^{M x^{3}} .
$$

This method to solve the BPS equation is called the moduli matrix formalism [3, 42]. The following $V$-transformations leave the physical fields $H$ in eq. (3.19) and $A_{i}$ and $\Sigma$ in eq. (3.18) unchanged.

$$
S\left(x^{1}, x^{2}, x^{3}\right) \rightarrow V(z) S\left(x^{1}, x^{2}, x^{3}\right), \quad H_{0}(z) \rightarrow V(z) H_{0}(z), \quad V(z) \in G L(2, \mathbb{C}),
$$

where elements of the $G L(2, \mathbb{C})$ matrix $V(z)$ are holomorphic functions in $z$. Therefore the moduli space of the monopole vortex complex becomes the moduli matrices divided by the $V$-equivalence relation.

The $\mathrm{U}(2)_{\mathrm{C}}$ gauge transformations act on $S^{-1}$ from the left as

$$
S^{-1} \rightarrow U_{\mathrm{C}} S^{-1}
$$

By defining $\mathrm{U}(2)_{\mathrm{C}}$ gauge invariant matrices $\Omega$ and $\Omega_{0}$,

$$
\Omega=S S^{\dagger}, \quad \Omega_{0}=H_{0} e^{2 M x^{3}} H_{0}^{\dagger},
$$

we can cast the remaining BPS equation (3.13) into the following master equation

$$
\frac{1}{g^{2} v^{2}}\left[4 \bar{\partial}\left(\partial \Omega \Omega^{-1}\right)+\partial_{3}\left(\partial_{3} \Omega \Omega^{-1}\right)\right]=\mathbf{1}_{2}-\Omega_{0} \Omega^{-1} .
$$

This master equation should be solved with the boundary condition

$$
\Omega \rightarrow \Omega_{0} \quad \text { as } \quad|\vec{x}| \rightarrow \infty
$$

The $\mathrm{U}(2)_{\mathrm{C}}$ gauge invariants $\Omega$ and $\Omega_{0}$ are covariant under the $V$-transformations.

\subsection{Vortex strings}

Before describing the monopole-vortex complex, let us first explain a simpler configuration of vortex strings without monopoles. Let us consider a vortex with unit vorticity corresponding to the Higgs field $H$ with a single zero. Since $S^{-1}$ is defined to have no singularities nor zeros, the zero should be placed either in upper-left or lower-right corner ${ }^{1}$ of the moduli matrix $H_{0}(z)$. Hence, in the $m \neq 0$ case, we have two different types of

\footnotetext{
${ }^{1}$ The zero can be placed only on the diagonal, otherwise one cannot solve the master equation.
} 

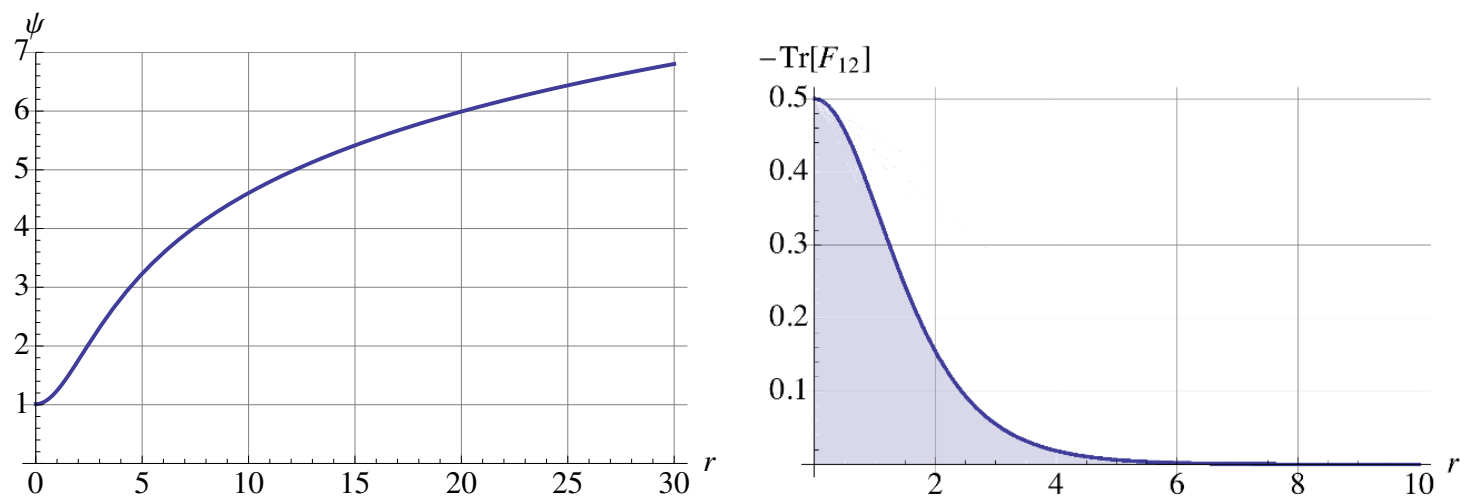

Figure 1. The left figure shows numerical solution $\psi(r)$ for eq. (3.28) and the right figure shows the magnetic field $\operatorname{Tr}\left[F_{12}\right]=-2 \partial \bar{\partial} \psi$ for the single vortex string (a cross-section of the flux tube at any $z$ ). We set the parameters by $g v=1$.

Abelian vortices (see also the argument around eq. (3.1)). When moduli matrix has a zero in the upper-left corner, we obtain

$$
H_{0}=\left(\begin{array}{ll}
z & 0 \\
0 & 1
\end{array}\right), \quad S=\left(\begin{array}{cc}
e^{\frac{\psi\left(x^{1}, x^{2}\right)}{2}} & 0 \\
0 & 1
\end{array}\right) e^{M x^{3}}
$$

which is called an N-vortex. ${ }^{2}$ Hereafter, we choose $\psi$ to be real by a gauge choice. One can easily see that the above matrices give us the following physical fields

$$
H_{\mathrm{N}-\text { vor }}=v\left(\begin{array}{cc}
e^{-\frac{\psi}{2}} z & 0 \\
0 & 1
\end{array}\right), \quad \Sigma=M, \quad \bar{A}_{\mathrm{N} \text {-vor }}=\left(\begin{array}{cc}
-\frac{i}{2} \bar{\partial} \psi & 0 \\
0 & 0
\end{array}\right), \quad A_{0,3}=0 .
$$

Then the magnetic field and gauge invariant $\Omega$ are given by

$$
F_{12}^{\mathrm{N}-\mathrm{vor}}=\left(\begin{array}{cc}
-2 \partial \bar{\partial} \psi & 0 \\
0 & 0
\end{array}\right), \quad \Omega^{\mathrm{N}-\mathrm{vor}}=\left(\begin{array}{cc}
e^{\psi+m x^{3}} & 0 \\
0 & e^{-m x^{3}}
\end{array}\right) .
$$

We find that the master equation (3.23) for the $\mathrm{N}$-vortex (3.25) reduces to the master equation for the Abelian vortex

$$
\frac{4}{g^{2} v^{2}} \partial \bar{\partial} \psi=1-|z|^{2} e^{-\psi}
$$

This equation has no known analytic solution, but can easily be solved numerically. The asymptotic behavior for $\psi$ is given by

$$
\psi \rightarrow \log |z|^{2}+q K_{0}(g v|z|), \quad \text { as } \quad|z| \rightarrow \infty,
$$

where $K_{0}$ is the modified Bessel function of the second kind and a constant $q$ can be obtained numerically. The function $\psi$ and magnetic field for the axially symmetric solution

\footnotetext{
${ }^{2}$ This terminology comes from the fact that the vortex is sitting at the north pole of the moduli space of the non-Abelian vortex for $m=0$.
} 
are obtained numerically and shown in figure 1 . Now it is easy to obtain the mass of the vortex as

$$
M_{\mathrm{vor}}=L_{3} \times v^{2} \int d x^{1} d x^{2} 2 \partial \bar{\partial} \psi=L_{3} \times 2 \pi v^{2},
$$

where $L_{3}$ is the length of the vortex string along the $x^{3}$ axis.

For later convenience, let us decompose the magnetic field $F_{12}$ in the $\mathrm{U}(2)_{\mathrm{C}}=\left(\mathrm{U}(1)_{\mathrm{C} 0} \times\right.$ $\left.\mathrm{SU}(2)_{\mathrm{C}}\right) / \mathbb{Z}_{2}$ gauge group into the field $F_{12}^{0}$ for the overall $\mathrm{U}(1)_{\mathrm{C} 0}$ and the field $F_{12}^{\Sigma}$ projected along the adjoint field $\Sigma$ (this is identical to the third component $\mathrm{U}(1)_{\mathrm{C} 3}$ of $\mathrm{SU}(2)_{\mathrm{C}}$ in the present case) as

$$
F_{i j}^{0}=\operatorname{Tr}\left[F_{i j} \frac{\mathbf{1}_{2}}{2}\right], \quad F_{i j}^{\Sigma}=\operatorname{Tr}\left[F_{i j} \frac{\Sigma}{m}\right] .
$$

We will call $F_{12}^{0}$ as Abelian magnetic field and $F_{12}^{\Sigma}$ as non-Abelain magnetic field. Note that these two magnetic fields are associated with $\left(\mathrm{U}(1)_{\mathrm{C} 0} \times \mathrm{U}(1)_{\mathrm{C} 3}\right) / \mathbb{Z}_{2} \subset \mathrm{U}(2)_{\mathrm{C}}$ (asymptotically) which is not broken by the adjoint scalar field $\Sigma=(\mathrm{m} / 2) \sigma_{3}$. A linear combination of these $\mathrm{U}(1)$ gauge symmetries is restored inside vortices. Therefore they are precisely the appropriate magnetic fields to measure the magnetic flux flowing to infinity through vortices. For the N-vortex, we obtain Abelian and non-Abelian magnetic fields as

$$
F_{12}^{0}=-\partial \bar{\partial} \psi, \quad F_{12}^{\Sigma}=-\partial \bar{\partial} \psi
$$

We see that only the sum $F_{12}^{0}+F_{12}^{\Sigma}$ (not the difference: $F_{12}^{0}-F_{12}^{\Sigma}=0$ does not play any role) has nonvanishing magnetic field inside the $\mathrm{N}$-vortex. This linear combination precisely corresponds to the restored $\mathrm{U}(1)$ gauge symmetry inside the N-vortex.

Another possibility to place the zero of the Higgs field is at the lower-right corner of the moduli matrix as

$$
H_{0}=\left(\begin{array}{ll}
1 & 0 \\
0 & z
\end{array}\right), \quad S=\left(\begin{array}{cc}
1 & 0 \\
0 & e^{\psi / 2}
\end{array}\right) e^{M x^{3}},
$$

where $\psi$ is the same function as the $\mathrm{N}$-vortex. We call this the S-vortex. Physical fields of the S-vortex are given by

$$
H_{\mathrm{S}-\mathrm{vor}}=v\left(\begin{array}{cc}
1 & 0 \\
0 & e^{-\frac{\psi}{2} z}
\end{array}\right), \quad \Sigma=M, \quad \bar{A}_{\mathrm{S} \text {-vor }}=\left(\begin{array}{cc}
0 & 0 \\
0 & -\frac{i}{2} \bar{\partial} \psi
\end{array}\right), \quad A_{0,3}=0 .
$$

The Abelian $F_{12}^{0}$ and non-Abelian $F_{12}^{3}$ magnetic fields of the S-vortex is given as

$$
F_{12}^{0}=-\partial \bar{\partial} \psi, \quad F_{12}^{\Sigma}=+\partial \bar{\partial} \psi
$$

We see that only the difference $F_{12}^{0}-F_{12}^{\Sigma}$ has nonvanishing magnetic field inside the Svortex. This linear combination is the restored $\mathrm{U}(1)$ gauge symmetry inside the S-vortex.

Note that the N-vortex and the S-vortex have the same Abelian magnetic fields but their non-Abelian magnetic fields are oriented in the opposite directions along the $x^{3}$ axis. The mass of the S-vortex equals to that of the N-vortex. 


\subsection{A monopole in the Higgs phase}

Now we are ready to understand the magnetic monopole. We connect the $\mathrm{N}$-vortex and S-vortex at a point on the $x^{3}$ axis. While the Abelian magnetic field $F_{12}^{0}$ can be smoothly connected, a non-trivial magnetic source is needed at the point to connect oppositely directed non-Abelian magnetic fields $F_{12}^{\Sigma}$. This source is nothing but a magnetic monopole. This configuration is simply described by the moduli matrix [42] with a complex moduli parameter $\phi$

$$
H_{0}(z)=\left(\begin{array}{cc}
z & 0 \\
-\phi & 1
\end{array}\right), \quad \phi=-e^{-m X_{m}-i \eta},
$$

where constant real parameters $X_{m}$ and $\eta$ correspond to the position and the phase of the monopole as we see immediately. In order to solve the master equation, a useful Ansatz for $\Omega$ was proposed with $\tau, \psi_{1}, \psi_{2}$ as functions of $x^{1}, x^{2}, x^{3}$ [40]

$$
\begin{aligned}
\Omega= & e^{M\left(X_{m}+i \eta / m\right)}\left(\begin{array}{cc}
1 & \tau\left(x^{1}, x^{2}, x^{3}\right) \\
0 & 1
\end{array}\right)\left(\begin{array}{cc}
e^{\psi_{1}\left(x^{1}, x^{2}, x^{3}\right)} & 0 \\
0 & e^{\psi_{2}\left(x^{1}, x^{2}, x^{3}\right)}
\end{array}\right) \\
& \times\left(\begin{array}{cc}
1 & 0 \\
\bar{\tau}\left(x^{1}, x^{2}, x^{3}\right) & 1
\end{array}\right) e^{M\left(X_{m}-i \eta / m\right)} .
\end{aligned}
$$

Even for the vortex configuration, the master equation cannot be solved analytically. The situation is the same for the monopole, and is even worse for the monopole-vortex system.

In what follows, we study the monopole configuration in the parameter region

$$
m \ll g v .
$$

It was found [40] that, to the first order in powers of

$$
\epsilon \equiv \frac{m}{g v},
$$

the master equation (3.23) is solved analytically with the Ansatz (3.37) as

$$
\begin{aligned}
\psi_{1}\left(x^{1}, x^{2}, x^{3}\right) & =\psi(z, \bar{z})-\log \left\{2 \cosh m\left(x^{3}-X_{m}\right)\right\}+\mathcal{O}\left(\epsilon^{2}\right), \\
\psi_{2}\left(x^{1}, x^{2}, x^{3}\right) & =\log \left\{2 \cosh m\left(x^{3}-X_{m}\right)\right\}+\mathcal{O}\left(\epsilon^{2}\right), \\
\tau\left(x^{1}, x^{2}, x^{3}\right) & =\frac{z e^{m\left(x^{3}-X_{m}\right)}}{2 \cosh m\left(x^{3}-X_{m}\right)}+\mathcal{O}\left(\epsilon^{2}\right),
\end{aligned}
$$

where $\psi(z, \bar{z})$ satisfies eq. (3.28). Using the Ansatz in eq. (3.37) with the solutions in eqs. (3.40)-(3.42), the physical fields can be cast into the following form after a gauge choice $^{3}$

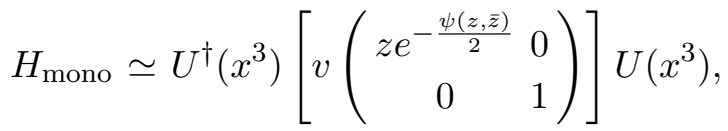

$$
\begin{aligned}
& \bar{A}_{\text {mono }} \simeq U^{\dagger}\left(x^{3}\right)\left(\begin{array}{cc}
-\frac{i}{2} \bar{\partial} \psi(z, \bar{z}) & 0 \\
0 & 0
\end{array}\right) U\left(x^{3}\right) \text {, }
\end{aligned}
$$

\footnotetext{
${ }^{3}$ In the paper [40], the configurations in the singular gauge are given.
} 
with

$$
\begin{aligned}
U\left(x^{3}\right) & =\frac{1}{\sqrt{1+\left|\phi\left(x^{3}\right)\right|^{2}}}\left(\begin{array}{cc}
1 & \bar{\phi}\left(x^{3}\right) \\
-\phi\left(x^{3}\right) & 1
\end{array}\right) \in \mathrm{SU}(2), \\
\phi\left(x^{3}\right) & =-\exp \left(m\left(x^{3}-X_{m}\right)-i \eta\right) .
\end{aligned}
$$

The structure of this peculiar solution will be explained in section 4. From now on we set $X_{m}=0$ and $\eta=0$ for simplicity. The third component of the gauge field $A_{3}$ and the adjoint field $\Sigma$ are given by

$$
\begin{aligned}
A_{3} & \simeq \frac{i m}{2} \operatorname{sech} m x^{3} U^{\dagger}\left(\begin{array}{cc}
0 & 1-z e^{-\frac{\psi}{2}} \\
\bar{z} e^{-\frac{\psi}{2}}-1 & 0
\end{array}\right) U, \\
\Sigma & \simeq \frac{m}{2} \operatorname{sech} m x^{3} U^{\dagger}\left(\begin{array}{cc}
-\sinh m x^{3} & z e^{-\frac{\psi}{2}} \\
\bar{z} e^{-\frac{\psi}{2}} & \sinh m x^{3}
\end{array}\right) U .
\end{aligned}
$$

Here $\simeq$ stands for the equality up to $\mathcal{O}\left(\epsilon^{2}\right)$ terms. This approximate solution has no unknown functions. Although no analytic solution for $\psi$ is known, it is the solution of the master equation (3.28) for the Abelian vortex, which we have already solved numerically.

Now we are ready to see the physical meaning of the monopole connecting the $\mathrm{N}$-vortex and S-vortex strings. The asymptotic behavior of $U\left(x^{3}\right)$ defined in eq. (3.46) is given by

$$
U\left(x^{3}\right) \rightarrow\left\{\begin{array}{ccc}
-i \sigma_{2} & \text { for } & x^{3} \rightarrow \infty \\
\mathbf{1}_{2} & \text { for } & x^{3} \rightarrow-\infty
\end{array} .\right.
$$

Therefore, the asymptotic behavior of $H$ and $\bar{A}$ are

$$
\begin{gathered}
H_{\text {mono }} \rightarrow\left\{\begin{array}{lll}
H_{\text {S-vor }} & \text { for } & x^{3} \rightarrow \infty \\
H_{\mathrm{N}-\text { vor }} & \text { for } & x^{3} \rightarrow-\infty
\end{array},\right. \\
\bar{A}_{\text {mono }} \rightarrow\left\{\begin{array}{lll}
\bar{A}_{\text {S-vor }} & \text { for } & x^{3} \rightarrow \infty \\
\bar{A}_{\mathrm{N} \text {-vor }} & \text { for } & x^{3} \rightarrow-\infty
\end{array}\right.
\end{gathered}
$$

The $2 \times 2$ matrices of the $\mathrm{U}(2)$ magnetic field are computed as

$$
\begin{aligned}
& F_{12} \simeq-2 \partial \bar{\partial} \psi U^{\dagger}\left(\begin{array}{ll}
1 & 0 \\
0 & 0
\end{array}\right) U \\
& F_{23} \simeq \frac{m}{2} e^{-\frac{1}{2} \psi} \operatorname{sech} m x^{3} U^{\dagger}\left(\begin{array}{cc}
0 & 1-z \partial \psi \\
1-\bar{z} \bar{\partial} \psi & 0
\end{array}\right) U, \\
& F_{31} \simeq \frac{i m}{2} e^{-\frac{1}{2} \psi} \operatorname{sech} m x^{3} U^{\dagger}\left(\begin{array}{cc}
0 & 1-z \partial \psi \\
\bar{z} \bar{\partial} \psi-1 & 0
\end{array}\right) U .
\end{aligned}
$$

Next, we compute the Abelian magnetic field $F_{i j}^{0}$ and the non-Abelian magnetic field $F_{i j}^{\Sigma}$ defined in eq. (3.31) to find

$$
F_{12}^{0} \simeq-\partial \bar{\partial} \psi, \quad F_{23}^{0} \simeq 0, \quad F_{31}^{0} \simeq 0,
$$


and

$$
\begin{aligned}
& F_{12}^{\Sigma} \simeq \partial \bar{\partial} \psi \tanh m x^{3}, \\
& F_{23}^{\Sigma} \simeq \frac{m}{4} \operatorname{sech}^{2} m x^{3} \partial_{1}\left(r^{2} e^{-\psi}\right), \\
& F_{31}^{\Sigma} \simeq \frac{m}{4} \operatorname{sech}^{2} m x^{3} \partial_{2}\left(r^{2} e^{-\psi}\right),
\end{aligned}
$$

where $r=\sqrt{\left(x^{1}\right)^{2}+\left(x^{2}\right)^{2}}$. The Abelian (overall $\mathrm{U}(1)$ ) magnetic field $F_{i j}^{0}$ is the same for the N-vortex and the S-vortex, whereas the non-Abelian magnetic field $F_{i j}^{\Sigma}$ is non-trivially changed from the N-vortex to the S-vortex. The monopole-vortex system has two scales

$$
L_{\mathrm{T}}=\frac{1}{g v}, \quad L_{\mathrm{L}}=\frac{1}{m} .
$$

As shown in eq. (3.29), the asymptotic behavior of $\psi$ is given by $\psi \sim \log r^{2}+q K_{0}\left(r / L_{\mathrm{T}}\right)$. Because of $K_{0}\left(r / L_{\mathrm{T}}\right) \sim \exp \left(-r / L_{\mathrm{T}}\right)$ for $r / L_{\mathrm{T}} \gg 1$, the scale $L_{\mathrm{T}}$ represents the transverse (orthogonal to the $x^{3}$ axis) size of the vortex string. On the other hand, $L_{\mathrm{L}}$ is another typical length scale within which the N-vortex is changed to the S-vortex. Namely, $L_{\mathrm{L}}$ is the longitudinal size of the magnetic monopole. Note that our approximation (3.38) is valid only for

$$
L_{\mathrm{L}} \gg L_{\mathrm{T}}
$$

Namely, the monopole in this regime is not spherically symmetric but has ellipsoidal shape. For this reason we call this solution a slender monopole. In figure 2, we show a contour of the topological charge density

$$
\mathcal{Q}_{m} \equiv \frac{1}{2 g} \epsilon_{i j k} \partial_{i} F_{j k}^{\Sigma}
$$

in order to visualize the slender monopole.

From eq. (3.11), the total energy of the static monopole is given by

$$
M_{\text {mono }}=\frac{2 m}{g^{2}} \int d^{3} x\left(\partial_{1} F_{23}^{\Sigma}+\partial_{2} F_{31}^{\Sigma}+\partial_{3} F_{12}^{\Sigma}\right)=\frac{4 \pi m}{g^{2}} .
$$

Note that the monopole contribution to the total energy is of order $\mathcal{O}\left(\epsilon^{2}\right)$, while that of the host vortex string is of order $\mathcal{O}(1)$. This can be seen as

$$
E_{\mathrm{tot}}=2 \pi v^{2} \times L_{\mathrm{L}}+\frac{4 \pi m}{g^{2}}=\frac{2 \pi v^{2}}{m}\left(1+2 \epsilon^{2}\right),
$$

where the first term corresponds to the mass of the vortex string of length $L_{\mathrm{L}}$.

One should note that all of the non-Abelian magnetic field lines of $\left(F_{23}^{\Sigma}, F_{31}^{\Sigma}, F_{12}^{\Sigma}\right)$ start from a point, as illustrated in the upper figure of figure 3. However, the Abelian magnetic field lines $\left(F_{23}^{0}, F_{31}^{0}, F_{12}^{0}\right)$ form trivially straight lines like a flux tube as depicted in the lower figure of figure 3 . 


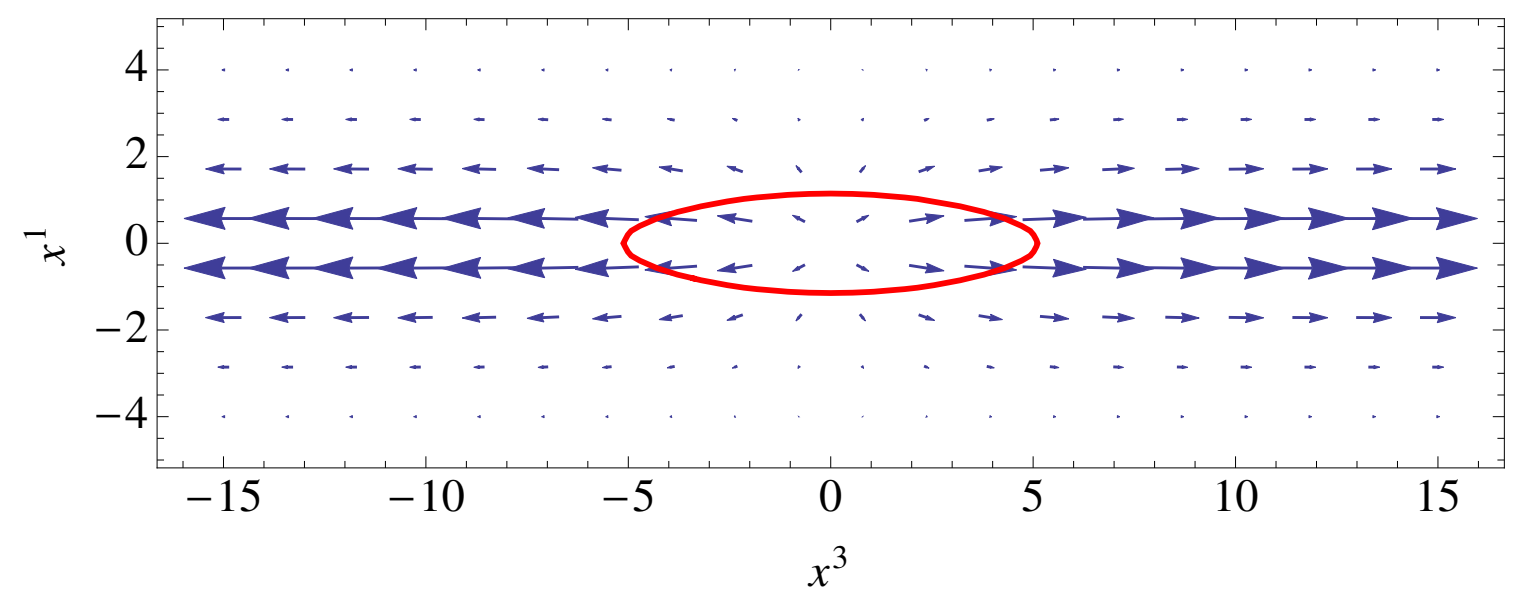

Figure 2. The slender magnetic monopole in the Higgs phase. The parameters are set as $g v=1$ and $m=1 / 5$. The red solid curve shows a contour of the topological charge density $\mathcal{Q}_{m}=0.05$. The vectors show the magnetic field of $\left(F_{12}^{\Sigma}, F_{23}^{\Sigma}\right)$. Length of the vector is proportional to norm of the magnetic field.

\section{Rigid-body approximation}

\subsection{Formalism}

In this section, we consider the same configuration, namely monopoles in the non-Abelian superconducting phase, from another perspective. We use a systematic expansion up to the next-to-leading order within an approximation, which we call the rigid-body approximation. As a result, we can understand the Ansatz and the analytic solutions of the previous section using a more systematic approach. Moreover, the results can be extended to consider time-dependent solutions as we discuss dynamics of monopoles in the next section. The transverse size of the vortex string $L_{\mathrm{T}}=1 /(g v)$ is associated with a large mass scale $g v$, and the longitudinal monopole size $L_{\mathrm{L}}=1 / \mathrm{m}$ is associated with a small mass scale. Therefore the condition $g v \gg m$ introduces hierarchal mass scales in the system: the thin vortexstring is generated at the high energy scale $\sim g v$, and the slender monopole is generated at the lower energy scale $\sim m$.

This picture allows us to understand the slender monopole as a kink in the $1+1 \mathrm{di}$ mensional theory on the vortex world-sheet [39]. Assuming

$$
\epsilon=\frac{m}{g v} \sim \frac{\partial_{\alpha}}{\partial_{i}} \ll 1, \quad(\alpha=0,3 \text { and } i=1,2),
$$

we expand the fields in power series of $\epsilon$

$$
\begin{array}{rlrl}
H & =H^{(0)}+H^{(2)}+\cdots, & \\
A_{i}=A_{i}^{(0)}+A_{i}^{(2)}+\cdots, & & (i=1,2), \\
A_{\alpha} & =A_{\alpha}^{(1)}+A_{\alpha}^{(3)}+\cdots, & & (\alpha=0,3), \\
\Sigma & =\Sigma^{(1)}+\Sigma^{(3)}+\cdots, &
\end{array}
$$



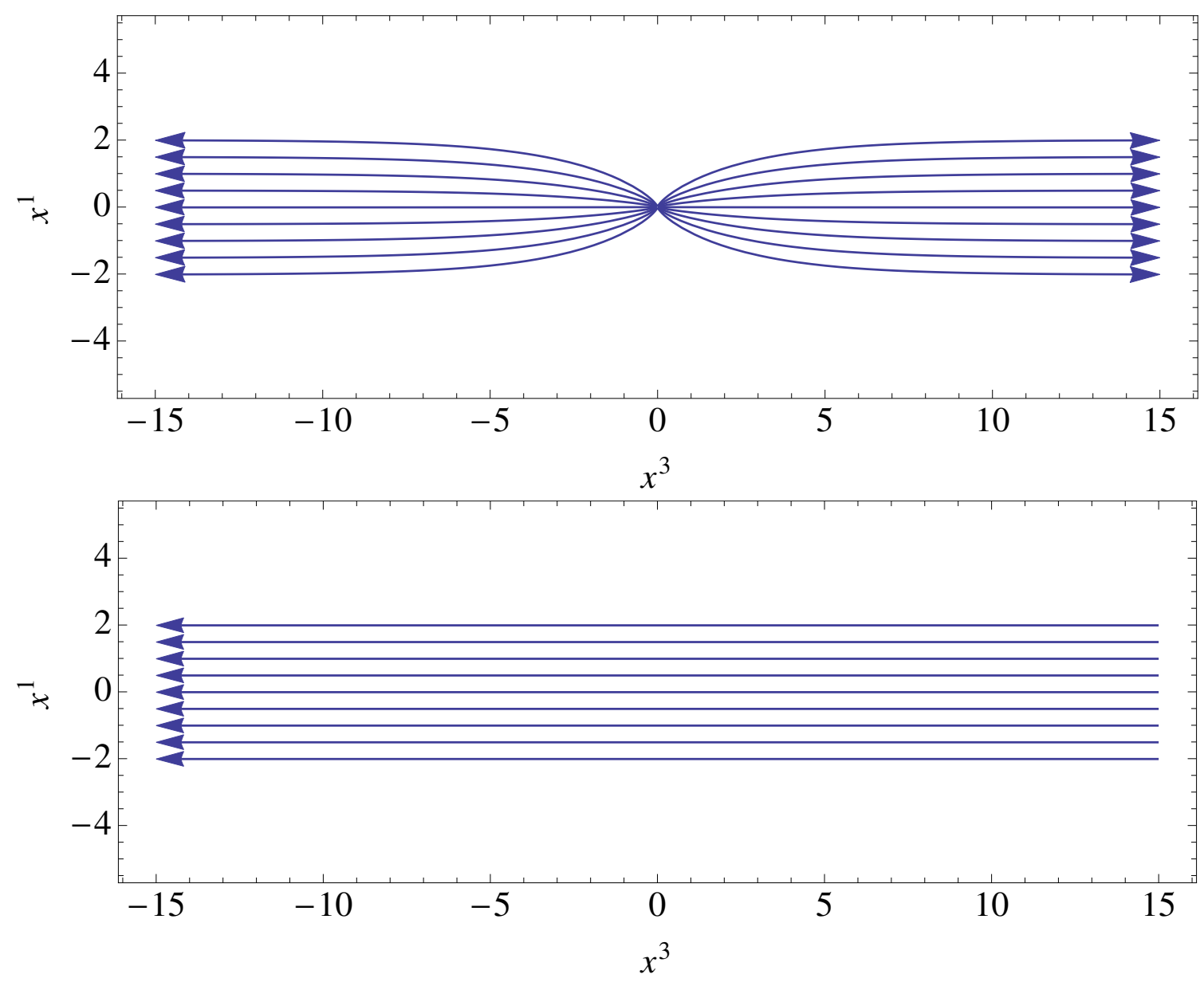

Figure 3. Stream line plots of the Abelian magnetic fields of $\left(F_{12}^{\Sigma}, F_{23}^{\Sigma}\right)$ (upper) and $\left(F_{12}^{0}, F_{23}^{0}\right)$ (lower). The parameters are the same as those for figure 2. We pick up the stream lines which pass the points $x^{1}=\{ \pm 2, \pm 1.5, \pm 1, \pm 0.5,0\}$ for $x^{3}= \pm 14$.

where the superscript $(n)$ indicates the $n$-th order in powers of $\epsilon$. Note that $H$ and $A_{i}$ start from the zeroth order because they are nontrivial in the background vortex-string configuration. On the other hand, since $A_{\alpha}$ and $\Sigma$ vanish in the background vortex-string configuration, they start from the first order.

We would like to solve the full equations of motion:

$$
\begin{gathered}
D_{0}^{2} H-D_{3}^{2} H-2(D \bar{D}+\bar{D} D) H+\frac{g^{2}}{2}\left(H H^{\dagger}-v^{2} \mathbf{1}_{2}\right) H=2 \Sigma H M-H M^{2}-\Sigma^{2} H, \\
\frac{2}{g^{2}} D_{0} F_{0 \bar{z}}-\frac{2}{g^{2}} D_{3} F_{3 \bar{z}}-\frac{4}{g^{2}} \bar{D} F_{z \bar{z}}+i\left(H(\bar{D} H)^{\dagger}-\bar{D} H H^{\dagger}\right)=-\frac{2 i}{g^{2}}[\Sigma, \bar{D} \Sigma] \\
\frac{2}{g^{2}} D_{3} F_{03}+\frac{4}{g^{2}}\left(D F_{0 \bar{z}}+\bar{D} F_{0 z}\right)+i\left(H\left(D_{0} H\right)^{\dagger}-D_{0} H H^{\dagger}\right)=-\frac{2 i}{g^{2}}\left[\Sigma, D_{0} \Sigma\right] \\
\frac{2}{g^{2}} D_{0} F_{03}+\frac{4}{g^{2}}\left(D F_{3 \bar{z}}+\bar{D} F_{3 z}\right)+i\left(H D_{3} H^{\dagger}-D_{3} H H^{\dagger}\right)=-\frac{2 i}{g^{2}}\left[\Sigma, D_{3} \Sigma\right] \\
-\frac{2}{g^{2}}\left(D_{0}^{2} \Sigma-D_{3}^{2} \Sigma-2 D \bar{D} \Sigma-2 \bar{D} D \Sigma\right)=(\Sigma H-H M) H^{\dagger}+H\left(H^{\dagger} \Sigma-M H^{\dagger}\right)
\end{gathered}
$$


Zero-th order: background vortex string for $\boldsymbol{m}=\mathbf{0}$. Retaining only the zero-th order fields in eqs. (4.2)-(4.5), we find the following zero-th order equations

$$
\begin{aligned}
2\left(D \bar{D} H^{(0)}+\bar{D} D H^{(0)}\right)-\frac{g^{2}}{2}\left(H^{(0)} H^{(0) \dagger}-v^{2} \mathbf{1}_{2}\right) H^{(0)} & =0 \\
-\frac{4}{g^{2}} \bar{D} F_{z \bar{z}}^{(0)}+i\left(H^{(0)}\left(\bar{D} H^{(0)}\right)^{\dagger}-\bar{D} H^{(0)} H^{(0) \dagger}\right) & =0 .
\end{aligned}
$$

The zero-th order solutions can be compactly expressed in the moduli matrix formalism as

$$
H^{(0)}=v S^{(0)-1} H_{0}(z), \quad \bar{A}^{(0)}=-i S^{(0)-1} \bar{\partial} S^{(0)},
$$

with the master equation for the vortex

$$
\frac{4}{g^{2} v^{2}} \bar{\partial}\left(\partial \Omega^{(0)} \Omega^{(0)-1}\right)=\mathbf{1}_{2}-\Omega_{0}^{(0)} \Omega^{(0)-1}, \quad \Omega^{(0)}=S^{(0)} S^{(0) \dagger}, \quad \Omega_{0}^{(0)}=H_{0}^{(0)} H_{0}^{(0) \dagger} .
$$

They are identical to the vortex equations (3.18) (3.19), and (3.23) except for the additional condition $M=0$. When $M=0$, the flavor symmetry is enhanced from $\mathrm{U}(1)_{\mathrm{F}}$ to $\mathrm{SU}(2)_{\mathrm{F}}$ and the symmetry of the vacuum becomes $\mathrm{SU}(2)_{\mathrm{C}+\mathrm{F}}$. A single vortex spontaneously breaks this symmetry to $\mathrm{U}(1)_{\mathrm{C}+\mathrm{F}}$. Therefore, the Nambu-Goldstone zero modes $\phi$ appear as a moduli

$$
\mathbb{C} P^{1}=\frac{\mathrm{SU}(2)_{\mathrm{C}+\mathrm{F}}}{\mathrm{U}(1)_{\mathrm{C}+\mathrm{F}}} \simeq S^{2} .
$$

By introducing the moduli parameter $\phi$ as an inhomogeneous coordinate of the moduli space $\mathbb{C} P^{1} \simeq S^{2}$, we can express the generic moduli matrix $H_{0}$ with the moduli parameter $\phi \in \mathbb{C}$ as a color-flavor $\mathrm{SU}(2)_{\mathrm{C}+\mathrm{F}}$ rotation of the $\mathrm{N}$-vortex solution together with an accompanying $V$-transformation as

$$
\begin{aligned}
H_{0}^{(0)} & =\left(\begin{array}{cc}
z & 0 \\
-\phi & 1
\end{array}\right)=V\left(\begin{array}{ll}
z & 0 \\
0 & 1
\end{array}\right) U, \\
S^{(0)} & =\left(\begin{array}{cc}
\frac{e^{\frac{\psi}{2}}+z|\phi|^{2}}{1+|\phi|^{2}} & \frac{\left(e^{\frac{\psi}{2}}-z\right) \bar{\phi}}{1+|\phi|^{2}} \\
-\phi & 1
\end{array}\right)=V\left(\begin{array}{cc}
e^{\frac{\psi}{2}} & 0 \\
0 & 1
\end{array}\right) U,
\end{aligned}
$$

with

$$
\begin{aligned}
& U=\frac{1}{\sqrt{1+|\phi|^{2}}}\left(\begin{array}{cc}
1 & \bar{\phi} \\
-\phi & 1
\end{array}\right) \in \mathrm{SU}(2)_{\mathrm{C}+\mathrm{F}} \\
& V=\frac{1}{\sqrt{1+|\phi|^{2}}}\left(\begin{array}{cc}
1 & -\bar{\phi} z \\
0 & 1+|\phi|^{2}
\end{array}\right)
\end{aligned}
$$

Here we need the $V$-transformation $V(z)$, in order for $H_{0}^{(0)}$ to be a holomorphic function of the moduli parameter $\phi$. The single vortex solution with the generic moduli $\phi$ can be obtained explicitly by inserting the $\mathrm{N}$-vortex solution $\psi$ into eqs. (4.17) and (4.13).

At the zero-th order in $\epsilon \ll 1$, we obtained a moduli parameter $\phi$ in eqs. (4.18) and (4.19) as a constant. However, our approximation allows the weak dependence of $\phi$ on 
$x^{0}, x^{3}$ from the beginning. Therefore, we should consider $\phi\left(x^{0}, x^{3}\right)$ to be a slowly varying function of $x^{0}, x^{3}$. Then, the vortex background configuration depends on $x^{0}$ and $x^{3}$ only through the moduli field.

$$
H^{(0)}\left(x^{1}, x^{2} ; \phi\left(x^{0}, x^{3}\right)\right), \quad A_{i}^{(0)}\left(x^{1}, x^{2} ; \phi\left(x^{0}, x^{3}\right)\right) \quad(i=1,2) .
$$

The various fields are then induced by this slowly varying $\phi\left(x^{0}, x^{3}\right)$, and are determined by the full equations of motion in eqs. (4.6)-(4.10). The determination of these is our task in the following.

Here one important point on the boundary condition is in order. In constructing the zero-th order solution, we need to use the boundary condition appropriate for $m \neq 0$ case even though the zero-th order equations of motion corresponds to the $m=0$ case. Otherwise, our power series expansion cannot work. Therefore the vortex moduli $\phi$ should tend to 0 or $\infty$ at asymptotic region $x^{3} \rightarrow \pm \infty$ : we need to choose the $\mathrm{N}$-vortex or the S-vortex at $x^{3} \rightarrow \pm \infty$ as the zero-th order solution. As a result, the slowly varying moduli field $\phi\left(x^{0}, x^{3}\right)$ interpolates the $\mathrm{N}$-vortex and/or the S-vortex at asymptotic regions $x^{3} \rightarrow \pm \infty$.

First order: Gauss's law constraints. Let us next solve the first order equations:

$$
\begin{aligned}
& \frac{4}{g^{2}}\left(D F_{\alpha \bar{z}}^{(1)}+\bar{D} F_{\alpha z}^{(1)}\right)+i\left(H^{(0)} D_{\alpha} H^{(0) \dagger}-D_{\alpha} H^{(0)} H^{(0) \dagger}\right)=0, \quad(\alpha=0,3), \\
& \frac{4}{g^{2}}(D \bar{D}+\bar{D} D) \Sigma^{(1)}-\left(\Sigma^{(1)} H^{(0)}-H^{(0)} M\right) H^{(0) \dagger}-H^{(0)}\left(H^{(0) \dagger} \Sigma^{(1)}-M H^{(0) \dagger}\right)=0 .
\end{aligned}
$$

We call these the Gauss's law constraints which determine $A_{\alpha}^{(1)}(\alpha=0,3)$ and $\Sigma^{(1)}$ for a given background vortex configuration in eq. (4.20) with a slowly varying moduli field $\phi\left(x^{0}, x^{3}\right)$. Moreover, the deformation of the vortex profile in $x^{1}, x^{2}$ plane arises from the higher order terms $H^{(2)}, H^{(4)}, \cdots$ by including massive modes and higher derivative corrections. In this paper, we consider up to the first order in $\epsilon$. Therefore the background vortex is given only by the zero-th order term $H^{(0)}$ with the slowly varying moduli field $\phi\left(x^{0}, x^{3}\right)$. Since the zero-th order fields $H^{(0)}$ and $A_{1,2}^{(0)}$ depend on $\phi\left(x^{0}, x^{3}\right)$ only through the flavor transformation $U \in \mathrm{SU}(2)_{\mathrm{F}}$, energy density of the background vortex string does not depend on $x^{0}, x^{3}$. In other words, the vortex string is treated as a rigid body. This is the reason why we call our approximation the rigid-body approximation.

While the $x^{0}, x^{3}$ dependence of $H$ and $A_{1,2}$ fields comes only through the $U$ flavor rotation, the fields $A_{0,3}$ and $\Sigma$ depend in addition on the $V$-transformation matrix (4.19) and also on the derivatives of both $U$ and $V$. This can be seen from the solution $[3,43,44]$ of first order equations (4.21)-(4.22) for an arbitrary $\phi\left(x^{0}, x^{3}\right)$

$$
\begin{aligned}
& A_{\alpha}^{(1)}=i\left[\left(\delta_{\alpha} S^{(0) \dagger}\right) S^{(0) \dagger-1}-S^{(0)-1} \delta_{\alpha}^{\dagger} S^{(0)}\right], \quad(\alpha=0,3), \\
& \Sigma^{(1)}=M+i\left[\left(\delta_{\phi} S^{(0) \dagger}\right) S^{(0) \dagger-1}-S^{(0)-1} \delta_{\phi}^{\dagger} S^{(0)}\right],
\end{aligned}
$$

with

$$
\delta_{\alpha}=\partial_{\alpha} \phi \frac{\delta}{\delta \phi}, \quad \delta_{\alpha}^{\dagger}=\partial_{\alpha} \bar{\phi} \frac{\delta}{\delta \bar{\phi}}, \quad \delta_{\phi}=-i m \phi \frac{\delta}{\delta \phi}, \quad \delta_{\phi}^{\dagger}=i m \bar{\phi} \frac{\delta}{\delta \bar{\phi}}
$$


The remaining task is to look for the appropriate configurations of $\phi\left(x^{0}, x^{3}\right)$ which minimize the energy of the solution. To this end, we plug $A_{\alpha}^{(1)}$ and $\Sigma^{(1)}$ into the original Lagrangian (2.1) and pick up terms up to the second order in $\epsilon$. After a tedious calculation, one obtains the following expression, where the $x^{1,2}$ and $x^{0,3}$ dependence are factorized as

$$
\mathcal{L}=-v^{2} F_{12}\left(x^{1}, x^{2}\right)+\frac{F\left(x^{1}, x^{2}\right)}{g^{2}} \times \frac{\left|\partial_{\alpha} \phi\left(x^{0}, x^{3}\right)\right|^{2}-m^{2}\left|\phi\left(x^{0}, x^{3}\right)\right|^{2}}{\left(1+\left|\phi\left(x^{0}, x^{3}\right)\right|^{2}\right)^{2}}+\mathcal{O}\left(\epsilon^{4}\right),
$$

where we ignore unessential total derivative terms. The prefactor in the second term depends on only $x^{1}$ and $x^{2}$ and it is given by

$$
F\left(x^{1}, x^{2}\right)=4 \partial \bar{\partial} \psi\left(x^{1}, x^{2}\right)
$$

Hence, in order to minimize the action to the second order, we need to find a stationary point of

$$
\mathcal{L}^{(2)}=\frac{F\left(x^{1}, x^{2}\right)}{g^{2}} \times \frac{\left|\partial_{\alpha} \phi\left(x^{0}, x^{3}\right)\right|^{2}-m^{2}\left|\phi\left(x^{0}, x^{3}\right)\right|^{2}}{\left(1+\left|\phi\left(x^{0}, x^{3}\right)\right|^{2}\right)^{2}}
$$

Since the prefactor $F\left(x^{1}, x^{2}\right)$ is determined at the zero-th order, our task is basically to solve the massive non-linear sigma model in two dimensions with the target space $\mathbb{C} P^{1}$. Note that the process here is essentially the same as a well known derivation of a low energy effective action in the moduli approximation. To obtain the effective action, one just needs to integrate the Lagrangian over $x^{1}$ and $x^{2}$. The resulting overall coefficient is $4 \pi=\int d x^{1} d x^{2} F\left(x^{1}, x^{2}\right)$ and thus

$$
\mathcal{L}_{\text {eff }}=\frac{4 \pi}{g^{2}} \frac{\left|\partial_{\alpha} \phi\left(x^{0}, x^{3}\right)\right|^{2}-m^{2}\left|\phi\left(x^{0}, x^{3}\right)\right|^{2}}{\left(1+\left|\phi\left(x^{0}, x^{3}\right)\right|^{2}\right)^{2}} .
$$

In summary, in order to solve the equations of motion to the first order, we just need to solve the equations of motion of the effective theory, and to plug the solution $\phi\left(x^{0}, x^{3}\right)$ into $H\left(x^{1}, x^{2} ; \phi\left(x^{0}, x^{3}\right)\right)$ and $A_{1,2}\left(x^{1}, x^{2} ; \phi\left(x^{0}, x^{3}\right)\right)$. The remaining fields $A_{0,3}^{(1)}\left(x^{0}, x^{3}\right)$ and $\Sigma^{(1)}\left(x^{0}, x^{3}\right)$ to the first order are obtained through eqs. (4.23) and (4.24).

For later convenience, let us introduce another parametrization of $\mathbb{C} P^{1}$ in terms of polar angles $0 \leq \Theta \leq \pi$ and $0 \leq \Phi \leq 2 \pi$ as

$$
\phi=-e^{i \Phi} \tan \frac{\Theta}{2}
$$

The effective Lagrangian is rewritten as

$$
\mathcal{L}_{\text {eff }}=\frac{\pi}{g^{2}}\left[\partial_{\alpha} \Theta \partial^{\alpha} \Theta+\sin ^{2} \Theta \partial_{\alpha} \Phi \partial^{\alpha} \Phi-m^{2} \sin ^{2} \Theta\right]
$$

The scalar potential $\left(\pi m^{2} / g^{2}\right) \sin ^{2} \Theta$ is minimized at $\Theta=0$ and $\Theta=\pi$. Clearly, these correspond to the N-vortex $(\phi=0)$ and the S-vortex $(\phi=\infty)$. 


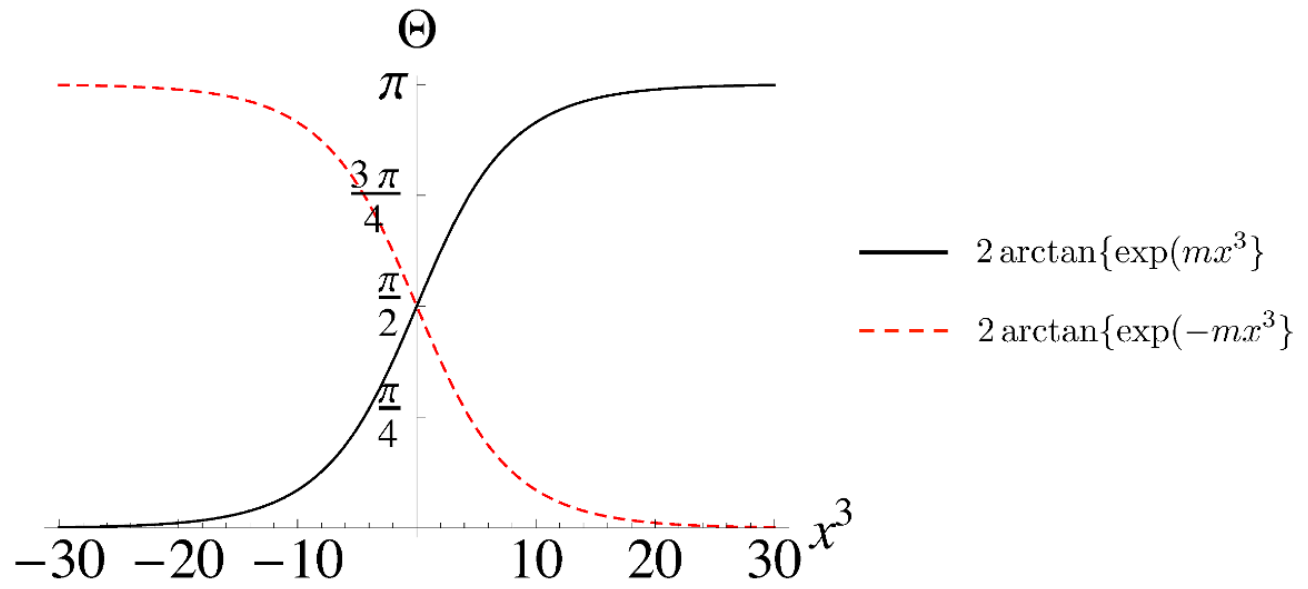

Figure 4. The sine-Gordon kink (black solid line) and anti-kink (red dashed line) for $m=1 / 5$.

\subsection{Monopole}

We are now ready to reconsider the slender magnetic monopole in the Higgs phase in our rigid-body approximation. Let us first look for an appropriate moduli configuration which minimizes the action in $1+3$ dimensions by solving the equations of motion in the low energy effective theory:

$$
\begin{aligned}
\partial_{\alpha} \partial^{\alpha} \Theta+\left(m^{2}-\partial_{\alpha} \Phi \partial^{\alpha} \Phi\right) \sin \Theta \cos \Theta & =0, \\
\partial^{\alpha}\left(\sin ^{2} \Theta \partial_{\alpha} \Phi\right) & =0 .
\end{aligned}
$$

eq. (4.33) admits a constant solution for $\Phi$, say $\Phi=\eta$. Throughout this work, we focus our attention to this class of solutions. Then the equation of motion reduces to the sine-Gordon equation. For static solutions it becomes

$$
-\Theta^{\prime \prime}+m^{2} \sin \Theta \cos \Theta=0,
$$

where the prime stands for the derivative in terms of $x^{3}$. The sine-Gordon model admits non-trivial topological excitations, kinks. The kinks interpolating $\Theta=0$ and $\Theta=\pi$ are given by

$$
\Theta=2 \arctan \exp \left( \pm m\left(x^{3}-X_{m}\right)\right) .
$$

The solution with the plus sign is the kink connecting $\Theta=0$ at $x^{3} \rightarrow-\infty$ and $\Theta=\pi$ at $x^{3} \rightarrow+\infty$, while that with minus sign is the anti-kink which connects $\Theta=\pi$ at $x^{3} \rightarrow-\infty$ and 0 at $x^{3} \rightarrow+\infty$, see figure 4 .

As is explained at the end of section 4.1, $\Theta=0$ corresponds to the $\mathrm{N}$-vortex and $\Theta=\pi$ corresponds to the S-vortex. Since the sine-Gordon kinks connect two different vortices, it should be a magnetic monopole. Now, we are ready to obtain the solution to the full $1+3$ dimensional equations of motion in eqs. (4.6)-(4.10) to the first order in $\epsilon$ according to the prescription given in the previous subsection. Firstly, the kink solution in terms of $\phi$ coordinate is

$$
\phi=-\exp \left( \pm m\left(x^{3}-X_{m}\right)-i \eta\right)
$$


where $X_{m}$ and $\eta$ are the moduli representing the position and phase of the monopole. ${ }^{4}$ From now on, we consider $X_{m}=\eta=0$ case for simplicity. Combining this with eqs. (4.13), (4.16), (4.17), and (4.18), we find

$$
\begin{aligned}
H^{(0)}\left(x^{1}, x^{2}, \phi\left(x^{3}\right)\right) & =v U^{\dagger}\left(x^{3}\right)\left(\begin{array}{cc}
z e^{-\frac{\psi}{2}} & 0 \\
0 & 1
\end{array}\right) U\left(x^{3}\right), \\
\bar{A}^{(0)}\left(x^{1}, x^{2}, \phi\left(x^{3}\right)\right) & =U^{\dagger}\left(x^{3}\right)\left(\begin{array}{cc}
-\frac{i}{2} \bar{\partial} \psi & 0 \\
0 & 0
\end{array}\right) U\left(x^{3}\right),
\end{aligned}
$$

with

$$
U\left(x^{3}\right)=\frac{1}{\sqrt{1+\left|\phi\left(x^{3}\right)\right|^{2}}}\left(\begin{array}{cc}
1 & \bar{\phi}\left(x^{3}\right) \\
-\phi\left(x^{3}\right) & 1
\end{array}\right) .
$$

Comparing these with the approximate solutions $H=H_{\text {mono }}$ and $\bar{A}=\bar{A}_{\text {mono }}$ in eqs. (3.43) and (3.44) obtained through the Ansatz in eq. (3.37), we find that $\left\{H_{\text {mono }}, \bar{A}_{\text {mono }}\right\}$ and $\left\{H^{(0)}, \bar{A}^{(0)}\right\}$ are identical (apart from sign choices in eq. (4.36) and the moduli parameters being set as $X_{\mathrm{m}}=0$ and $\left.\eta=0\right)$. Furthermore, plugging $S^{(0)}$

$$
S^{(0)}=\left(\begin{array}{cc}
\frac{e^{\frac{\psi}{2}}+z|\phi|^{2}}{1+|\phi|^{2}} & \frac{\left(e^{\frac{\psi}{2}}-z\right) \bar{\phi}}{1+|\phi|^{2}} \\
-\phi & 1
\end{array}\right), \quad \phi=-\exp \left( \pm m x^{3}\right)
$$

into the solutions $A_{\alpha}^{(1)}$ and $\Sigma^{(1)}$ given in eqs. (4.23) and (4.24), we obtain the induced fields

$$
\begin{aligned}
A_{3} & \simeq \pm \frac{i m}{2} \operatorname{sech} m x^{3} U^{\dagger}\left(x^{3}\right)\left(\begin{array}{cc}
0 & 1-z e^{-\frac{\psi}{2}} \\
\bar{z} e^{-\frac{\psi}{2}}-1 & 0
\end{array}\right) U\left(x^{3}\right), \\
\Sigma & \simeq \frac{m}{2} \operatorname{sech} m x^{3} U^{\dagger}\left(x^{3}\right)\left(\begin{array}{cc}
\mp \sinh m x^{3} & z e^{-\frac{\psi}{2}} \\
\bar{z} e^{-\frac{\psi}{2}} & \pm \sinh m x^{3}
\end{array}\right) U\left(x^{3}\right) .
\end{aligned}
$$

The configurations with the upper sign are exactly identical to those given in eqs. (3.47) and (3.48). In this way, we can interpret the approximate solution of the slender monopole given in section 3.3 as identical to the solution in the rigid-body approximation. The electric fields are the same as before:

$$
F_{12}^{0} \simeq-\partial \bar{\partial} \psi, \quad F_{23}^{0} \simeq 0, \quad F_{31}^{0} \simeq 0,
$$

and the sign of the magnetic fields depends on the sign choices of the moduli field in eq. (4.36)

$$
\begin{aligned}
& B_{3}^{\Sigma}=F_{12}^{\Sigma} \simeq \pm \partial \bar{\partial} \psi \tanh m x^{3}, \\
& B_{1}^{\Sigma}=F_{23}^{\Sigma} \simeq \pm \frac{m}{4} \partial_{1}\left(r^{2} e^{-\psi}\right) \operatorname{sech}^{2} m x^{3},
\end{aligned}
$$

\footnotetext{
${ }^{4}$ The moduli space of a monopole in the Higgs phase is $\mathbb{R} \times S^{1}$. In contrast, the moduli space of the usual $\mathrm{SU}(2)$ 't Hooft-Polyakov monopole in the Coulomb phase is $\mathbb{R}^{3} \times S^{1}$. The exponent $D$ of $\mathbb{R}^{D}$ corresponds to the spatial dimensions where the monopole can freely move. Including the position moduli of the host vortex string $\mathbb{R}^{2}$, the total moduli spaces of the monopole in the both phases coincide [45].
} 


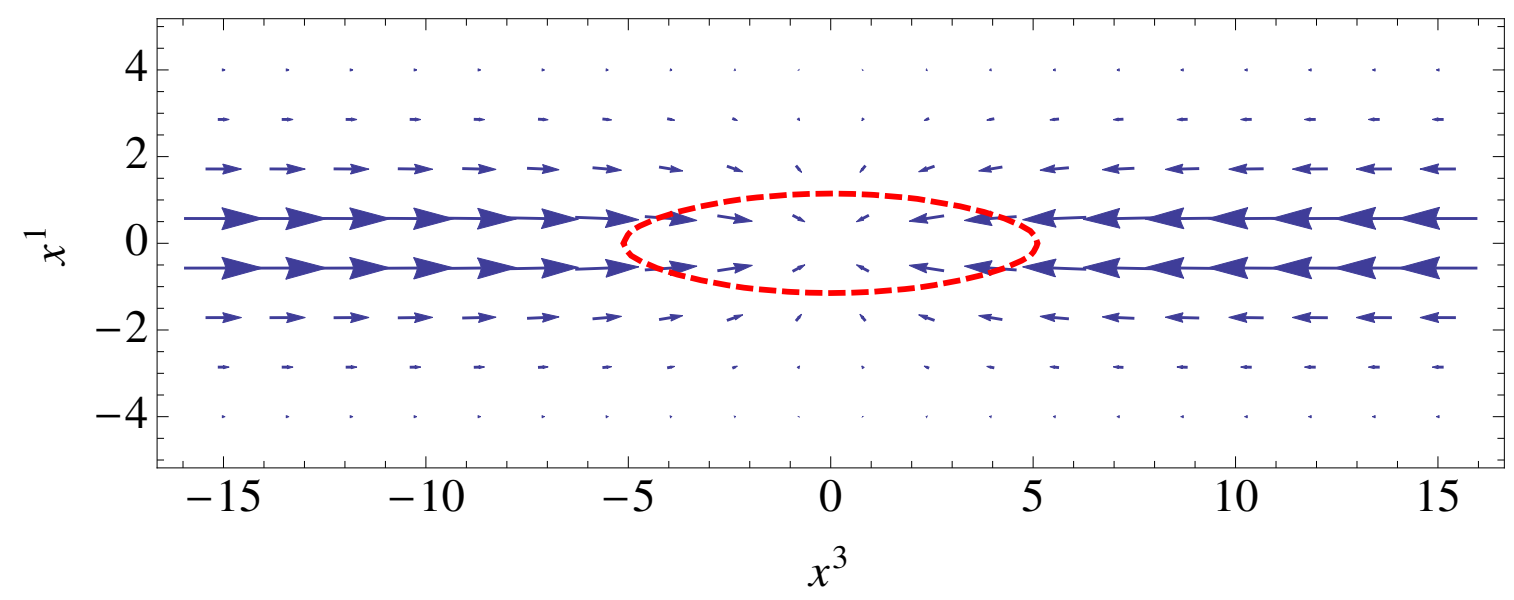

Figure 5. The slender magnetic anti-monopole in the Higgs phase. The parameters are set as $g v=1$ and $m=1 / 5$. The red dashed curve shows a contour of the topological charge density $\mathcal{Q}_{m}=-0.05$. The vectors show the magnetic field of $\left(F_{12}^{\Sigma}, F_{23}^{\Sigma}\right)$. Length of the vector is proportional to norm of the magnetic field.

$$
B_{2}^{\Sigma}=F_{31}^{\Sigma} \simeq \pm \frac{m}{4} \partial_{2}\left(r^{2} e^{-\psi}\right) \operatorname{sech}^{2} m x^{3}
$$

The new solution with the lower sign connects the N-vortex as $x^{3} \rightarrow+\infty$ and the S-vortex as $x^{3} \rightarrow-\infty$, which is opposite to the configuration with upper sign. The corresponding monopole has the magnetic field $F_{i j}^{\Sigma}$ pointing toward monopole, namely it is an anti-monopole in the Higgs phase. We show the configuration in figure 5.

Magnetic charges of the above solutions can be easily calculated

$$
Q_{\mathrm{m}}=\frac{1}{g} \int d^{3} x \operatorname{div} \vec{B}^{\Sigma}=\frac{1}{g}\left[\int_{x^{3} \rightarrow \infty} d x^{1} d x^{2}( \pm \partial \bar{\partial} \psi)-\int_{x^{3} \rightarrow-\infty} d x^{1} d x^{2}(\mp \partial \bar{\partial} \psi)\right]= \pm \frac{2 \pi}{g},
$$

where we used $\int d x^{1} d x^{2} \partial \bar{\partial} \psi=\pi$ and $r^{2} e^{-\psi} \rightarrow 1$ as $r \rightarrow \infty$. Here the factor $1 / g$ is needed due to our notation that the gauge coupling is absorbed in the gauge field, see eqs. (2.3)(2.5). This magnetic charge precisely coincides with one of the 't Hooft-Polyakov monopole in the Coulomb phase [39].

\section{Dynamics of slender monopoles and anti-monopoles}

\subsection{Brief summary of the rigid body approximation}

So far, we have studied the static single slender (anti-)monopole in the rigid-body approximation. Now we come to the main point of this paper, dynamics of the slender monopoles and anti-monopoles. To utilize the rigid-body approximation fully, let us summarize the result of the approximation in a compact form

$$
\begin{gathered}
H \approx H^{(0)}=v U^{\dagger}\left(x^{0}, x^{3}\right)\left(\begin{array}{cc}
z e^{-\frac{\psi}{2}} & 0 \\
0 & 1
\end{array}\right) U\left(x^{0}, x^{3}\right), \\
\bar{A} \approx \bar{A}^{(0)}=U^{\dagger}\left(x^{0}, x^{3}\right)\left(\begin{array}{cc}
-\frac{i}{2} \bar{\partial} \psi & 0 \\
0 & 0
\end{array}\right) U\left(x^{0}, x^{3}\right),
\end{gathered}
$$




$$
\begin{aligned}
A_{\alpha} & \approx A_{\alpha}^{(1)}=i\left[\left(\delta_{\alpha} S^{(0) \dagger}\right) S^{(0) \dagger-1}-S^{(0)-1} \delta_{\alpha}^{\dagger} S^{(0)}\right] \\
\Sigma & \approx \Sigma^{(1)}=M+i\left[\left(\delta_{\phi} S^{(0) \dagger}\right) S^{(0) \dagger-1}-S^{(0)-1} \delta_{\phi}^{\dagger} S^{(0)}\right],
\end{aligned}
$$

with

$$
\begin{aligned}
U\left(x^{0}, x^{3}\right) & =\frac{1}{\sqrt{1+\left|\phi\left(x^{0}, x^{3}\right)\right|^{2}}}\left(\begin{array}{cc}
1 & \bar{\phi}\left(x^{0}, x^{3}\right) \\
-\phi\left(x^{0}, x^{3}\right) & 1
\end{array}\right), \\
S^{(0)}= & \left(\begin{array}{cc}
\frac{e^{\frac{\psi}{2}}+z\left|\phi\left(x^{0}, x^{3}\right)\right|^{2}}{1+\left|\phi\left(x^{0}, x^{3}\right)\right|^{2}} & \frac{\left(e^{\frac{\psi}{2}}-z\right) \bar{\phi}\left(x^{0}, x^{3}\right)}{1+\left|\phi\left(x^{0}, x^{3}\right)\right|^{2}} \\
-\phi\left(x^{0}, x^{3}\right) & 1
\end{array}\right) .
\end{aligned}
$$

The moduli field $\phi\left(x^{0}, x^{3}\right)$

$$
\phi\left(x^{0}, x^{3}\right)=-e^{i \Phi\left(x^{0}, x^{3}\right)} \tan \frac{\Theta\left(x^{0}, x^{3}\right)}{2},
$$

should be a solution of the equations of motion

$$
\begin{aligned}
\partial_{\alpha} \partial^{\alpha} \Theta+\left(m^{2}-\partial_{\alpha} \Phi \partial^{\alpha} \Phi\right) \sin \Theta \cos \Theta & =0, \\
\partial^{\alpha}\left(\sin ^{2} \Theta \partial_{\alpha} \Phi\right) & =0 .
\end{aligned}
$$

\subsection{A dictionary: mapping onto the sine-Gordon model}

In the following, we fully make use of the similarity between our system and the sine-Gordon model. Let us denote another choice of the range of angles as

$$
\tilde{\Theta} \in \mathbb{R}(\bmod 2 \pi), \quad \tilde{\Phi} \in[0, \pi),
$$

to parametrize the $\mathbb{C} P^{1}$ moduli $\phi$

$$
\phi\left(x^{0}, x^{3}\right)=-e^{i \tilde{\Phi}\left(x^{0}, x^{3}\right)} \tan \frac{\tilde{\Theta}\left(x^{0}, x^{3}\right)}{2} .
$$

The equations of motion for $\tilde{\Theta}, \tilde{\Phi}$ are the same as those for $\Theta, \Phi$. Therefore, $\tilde{\Phi}=$ const. is a solution, to which we restrict ourselves in the following. Without loss of generality, the value of the constant $\Phi$ can be chosen as

$$
\tilde{\Phi}=0 .
$$

Then the equation of motion for $\tilde{\Theta}$ is reduced to

$$
\partial_{\alpha} \partial^{\alpha} \tilde{\Theta}+m^{2} \sin \tilde{\Theta} \cos \tilde{\Theta}=0, \quad \tilde{\Theta} \in \mathbb{R}(\bmod 2 \pi) .
$$

This is nothing but the sine-Gordon equation with a periodicity $\pi$ in $1+1$ dimensions.

Now we can compute all field configurations in $1+3$ dimensions with the help of the sine-Gordon field $\tilde{\Theta}$

$$
F_{12}^{0}=-\partial \bar{\partial} \psi, \quad F_{23}^{0}=F_{31}^{0}=F_{01}^{0}=F_{02}^{0}=F_{03}^{0}=0,
$$


and

$$
\begin{aligned}
& F_{12}^{\Sigma}=-\partial \bar{\partial} \psi \cos \tilde{\Theta}, \\
& F_{23}^{\Sigma}=\frac{1}{4} \partial_{1}\left(r^{2} e^{-\psi}\right) \partial_{3} \tilde{\Theta} \sin \tilde{\Theta}, \\
& F_{31}^{\Sigma}=\frac{1}{4} \partial_{2}\left(r^{2} e^{-\psi}\right) \partial_{3} \tilde{\Theta} \sin \tilde{\Theta}, \\
& F_{01}^{\Sigma}=\frac{1}{4} \partial_{2}\left(r^{2} e^{-\psi}\right) \partial_{0} \tilde{\Theta} \sin \tilde{\Theta}, \\
& F_{02}^{\Sigma}=-\frac{1}{4} \partial_{1}\left(r^{2} e^{-\psi}\right) \partial_{0} \tilde{\Theta} \sin \tilde{\Theta}, \\
& F_{03}^{\Sigma}=0 .
\end{aligned}
$$

Here we define Abelian and non-Abelian electric fields in the same sprit as in eq. (3.31)

$$
F_{0 i}^{0}=\operatorname{Tr}\left[F_{0 i} \frac{\mathbf{1}_{2}}{2}\right], \quad F_{0 i}^{\Sigma}=\operatorname{Tr}\left[F_{0 i} \frac{\Sigma}{m}\right] .
$$

Note that the electric field and magnetic field are orthogonal

$$
\epsilon^{i j k} F_{i j}^{\Sigma} F_{0 k}^{\Sigma}=0 .
$$

Therefore, there is no energy dissipation. This is the $1+3$-dimensional manifestation of the well-known special feature of the sine-Gordon dynamics: there is no dissipation of energy in multi-kink and anti-kink dynamics because of the infinite number of conservation law associated with the integrable sine-Gordon theory in $1+1$ dimensions.

The Hamiltonian density is decomposed into two parts: the energy density of the rigid vortex-string $\mathcal{H}_{\text {vortex }}^{(0)}$ and that of the dressed monopole $\mathcal{H}_{\text {dress }}^{(2)}$

$$
\mathcal{H}=\mathcal{H}_{\text {vortex }}^{(0)}+\mathcal{H}_{\text {dress }}^{(2)}+\mathcal{O}\left(\epsilon^{4}\right) .
$$

Note that the monopole contribution in our regime is of order $\mathcal{O}\left(\epsilon^{2}\right)$ as explained in eq. (3.63). The rigid vortex-string Hamiltonian density does not depend on $x^{0}$ and $x^{3}$

$$
\begin{aligned}
\mathcal{H}_{\mathrm{vortex}}^{(0)}= & \operatorname{Tr}\left[\frac{1}{g^{2}}\left(F_{12}^{(0)}\right)^{2}+\left(D_{i} H\right)^{(0)}\left(D_{i} H\right)^{(0) \dagger}+\frac{g^{2}}{4}\left(H^{(0)} H^{(0) \dagger}-v^{2} \mathbf{1}_{2}\right)^{2}\right] \\
= & \operatorname{Tr}\left[\frac{1}{g^{2}}\left(F_{12}^{(0)}-\frac{g^{2}}{2}\left(H^{(0)} H^{(0) \dagger}-v^{2} \mathbf{1}_{2}\right)\right)^{2}+4(\bar{D} H)^{(0)}(\bar{D} H)^{(0) \dagger}\right. \\
& \left.-v^{2} F_{12}^{(0)}+i\left\{\partial_{1}\left(H^{(0)}\left(D_{2} H\right)^{(0) \dagger}\right)-\partial_{2}\left(H^{(0)}\left(D_{1} H\right)^{(0) \dagger}\right)\right\}\right] \\
= & 2 v^{2} \mathcal{V} .
\end{aligned}
$$

where we used the master equation (3.28) to reach the last expression and defined a vortex energy density

$$
\mathcal{V}\left(x^{1}, x^{2}\right)=\partial \bar{\partial} \psi-\frac{4}{g^{2} v^{2}}(\partial \bar{\partial})^{2} \psi
$$

The first term in the right-hand side is the topological term while the second one is a total derivative which does not contribute to the total vortex energy. In deriving eq. (5.24), we 
have used the same identities in eqs. (3.6)-(3.9). The dressed Hamiltonian density which depends on $x^{0}$ and $x^{3}$ is given by

$$
\begin{aligned}
\mathcal{H}_{\mathrm{dress}}^{(2)} & =\operatorname{Tr}\left[\frac{1}{g^{2}}\left\{\left(F_{23}^{(1)}\right)^{2}+\left(F_{31}^{(1)}\right)^{2}+\left(F_{01}^{(1)}\right)^{2}+\left(F_{02}^{(1)}\right)^{2}+\left(D_{1} \Sigma^{(1)}\right)^{2}+\left(D_{2} \Sigma^{(1)}\right)^{2}\right\}\right. \\
& \left.+D_{0} H^{(0)}\left(D_{0} H^{(0)}\right)^{\dagger}+D_{3} H^{(0)}\left(D_{3} H^{(0)}\right)^{\dagger}+\left(\Sigma^{(1)} H^{(0)}-H^{(0)} M\right)\left(\Sigma^{(1)} H^{(0)}-H^{(0)} M\right)^{\dagger}\right] \\
& =\frac{\mathcal{V}}{g^{2}}\left(\left(\partial_{0} \tilde{\Theta}\right)^{2}+\left(\partial_{3} \tilde{\Theta}\right)^{2}+m^{2} \sin ^{2} \tilde{\Theta}\right),
\end{aligned}
$$

where we used the master equation (3.28).

Let us also add the topological charge density (3.61) in our dictionary

$$
\mathcal{Q}_{\mathrm{m}}=\frac{\mathcal{V}}{g} \partial_{3} \tilde{\Theta} \sin \tilde{\Theta}
$$

From this expression, one can easily compute the magnetic charge as

$$
Q_{\mathrm{m}}=\int d^{3} x \mathcal{Q}_{\mathrm{m}}=\frac{1}{g} \int d x^{1} d x^{2} \mathcal{V} \int d x^{3} \partial_{3} \tilde{\Theta} \sin \tilde{\Theta}=\frac{\pi}{g}[-\cos \tilde{\Theta}]_{x^{3}=-\infty}^{x^{3}=+\infty} .
$$

Here we used $\int d x^{1} d x^{2} \partial \bar{\partial} \psi=\pi$. As a check, one can compute the energy of the magnetic monopoles for the solutions given in eq. (4.35)

$$
Q_{\mathrm{m}}= \pm \frac{2 \pi}{g}
$$

Similarly, one may introduce a electric charge density by

$$
\mathcal{Q}_{\mathrm{e}}=\frac{1}{g} \partial^{i} F_{0 i}^{\Sigma}
$$

But this is identically zero for any $\tilde{\Theta}\left(x^{0}, x^{3}\right)$. This matches with a naive intuition that the fixed azimuthal angle $\tilde{\Phi}$ does not generate any electric charges. Note, however, that this does not mean the electric fields themselves are zeros. One can easily find that rotation of $\vec{E}^{\Sigma}=\left(F_{10}^{\Sigma}, F_{20}^{\Sigma}, F_{30}^{\Sigma}\right)$ are non-zero.

$$
\left(\vec{\nabla} \times \vec{E}^{\Sigma}\right)_{3}=-\frac{4}{g^{2} v^{2}}(\partial \bar{\partial})^{2} \psi \partial_{0} \tilde{\Theta} \sin \tilde{\Theta} .
$$

The other components are of higher order, so we ignore them.

\subsection{Two different species of slender monopoles}

The zenith angle $\tilde{\Theta}$ takes values between 0 and $2 \pi(=\mathbb{R} \bmod 2 \pi)$, and the sine-Gordon equation (5.13) is periodic with a period $\pi$. Therefore, there exist two sine-Gordon kinks: the one interpolates from 0 to $\pi$ as $x^{3}=-\infty \rightarrow+\infty$, and the other interpolates from $\pi$ to $2 \pi$ as $x^{3}=-\infty \rightarrow+\infty$. Here we need to pay some attention to our terminology in translating the sine-Gordon kinks into monopoles in $1+3$ dimensions. Although these two configurations are both to be called kinks in the sense of the sine-Gordon model, the former 

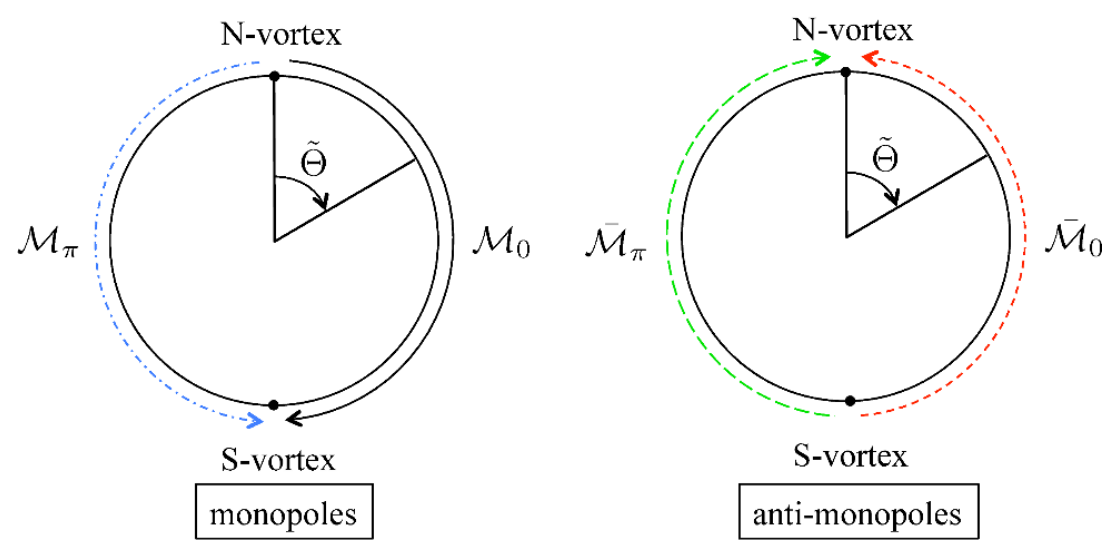

S-vortex

anti-monopoles

$\tilde{\Theta}$

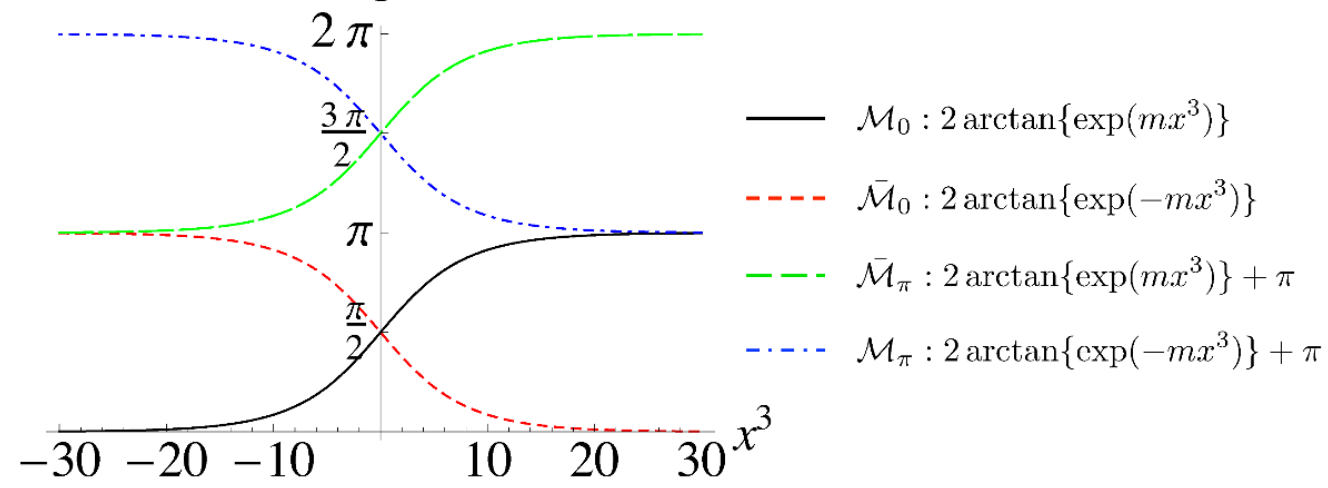

Figure 6. The slender monopole $\mathcal{M}_{0}$ (black solid line) corresponds to the sine-Gordon kink $(0$ to $\pi$ ), and another kind of the slender monopole $\mathcal{M}_{\pi}$ (blue dash-dotted line) to the sine-Gordon anti-kink $(2 \pi$ to $\pi)$. The slender anti-monopole $\overline{\mathcal{M}}_{0}$ (red dotted line) corresponds to the sineGordon anti-kink ( $\pi$ to 0 ), and another kind of slender anti-monopole $\overline{\mathcal{M}}_{\pi}$ (green dashed line) to the sine-Gordon kink ( $\pi$ to $2 \pi$ ).

connects the N-vortex and S-vortex from left to right, while the latter connects them from right to left. Namely, the former kink is the monopole (denoted as $\mathcal{M}_{0}$ ) and the latter kink is the anti-monopole $\left(\overline{\mathcal{M}}_{\pi}\right)$. Similarly, the anti-kink interpolating from $\pi$ to 0 as $x^{3}=-\infty \rightarrow$ $+\infty$ is the anti-monopole $\left(\overline{\mathcal{M}}_{0}\right)$, while the other anti-kink interpolating from $2 \pi$ to $\pi$ as $x^{3}=$ $-\infty \rightarrow+\infty$ is the monopole $\left(\mathcal{M}_{\pi}\right)$. Correspondence between the sine-Gordon (anti-)kinks and the slender (anti-)monopoles are depicted in figure 6 . The configurations are given by

$$
\begin{aligned}
& \mathcal{M}_{0}: \quad \tilde{\Theta}=2 \arctan \exp \left(m x^{3}\right)+2 n \pi, \\
& \overline{\mathcal{M}}_{0}: \tilde{\Theta}=2 \arctan \exp \left(-m x^{3}\right)+2 n \pi, \\
& \mathcal{M}_{\pi}: \quad \tilde{\Theta}=2 \arctan \exp \left(-m x^{3}\right)+(2 n+1) \pi, \\
& \overline{\mathcal{M}}_{\pi}: \quad \tilde{\Theta}=2 \arctan \exp \left(m x^{3}\right)+(2 n+1) \pi,
\end{aligned}
$$

with $n$ being an integer.

An advantage of mapping the slender monopoles in the non-Abelian superconductor onto the sine-Gordon system is that the interactions between the slender monopoles and 
anti-monopoles can be easily found. For instance, forces between the sine-Gordon kink and anti-kink have been obtained $[1,48]$ assuming large separation $R$. We just need to translate them to the interactions of slender monopoles in the non-Abelian gauge theory. The slender monopole and anti-monopole of the same kind $\left(\mathcal{M}_{0}\right.$ and $\overline{\mathcal{M}}_{0}$, or $\mathcal{M}_{\pi}$ and $\left.\overline{\mathcal{M}}_{\pi}\right)$ at large separations $R$ exert an attractive force each other

$$
\mathcal{F}\left(\mathcal{M}_{0}: \overline{\mathcal{M}}_{0}\right)=\mathcal{F}\left(\mathcal{M}_{\pi}: \overline{\mathcal{M}}_{\pi}\right)=-4 m^{2} \exp (-m R)
$$

On the other hand, the interaction between the monopole and anti-monopole of the different kind $\left(\mathcal{M}_{0}\right.$ and $\overline{\mathcal{M}}_{\pi}$ or $\mathcal{M}_{\pi}$ and $\left.\overline{\mathcal{M}}_{0}\right)$ at large separations $R$ is a repulsion

$$
\mathcal{F}\left(\mathcal{M}_{0}: \overline{\mathcal{M}}_{\pi}\right)=\mathcal{F}\left(\mathcal{M}_{\pi}: \overline{\mathcal{M}}_{0}\right)=+4 m^{2} \exp (-m R)
$$

This is because both of $\mathcal{M}_{0}$ and $\overline{\mathcal{M}}_{\pi}\left(\mathcal{M}_{\pi}\right.$ and $\left.\overline{\mathcal{M}}_{0}\right)$ are kinks (anti-kinks) with half windings $(\Delta \Theta=\pi)$ as solitons in the sine-Gordon system. The exponential force is in sharp contrast to the Coulomb force between monopoles in the unbroken phase. Since the gauge fields become massive in the superconducting phase, the interaction between monopoles becomes the Yukawa type which decreases exponentially with the Compton wave length $1 / m$ of the massive particle.

\subsection{Magnetic meson}

It is well-known that the sine-Gordon model admits a bound state of kink and anti-kink, the so-called breather solution. In our case, it is nothing but a bound state of the slender monopole and anti-monopole, which we call the magnetic meson. The configuration is given by

$$
\tilde{\Theta}\left(x^{0}, x^{3}\right)=2 \arctan \left(\frac{\eta \sin \omega x^{0}}{\cosh \eta \omega x^{3}}\right), \quad \eta=\sqrt{\frac{m^{2}}{\omega^{2}}-1}, \quad \omega<m,
$$

where $\omega$ is the frequency and $(\eta \omega)^{-1}=1 / \sqrt{m^{2}-\omega^{2}}$ is the typical size of the magnetic meson. The mass of the meson depends on $\omega$ as

$$
M_{\text {meson }}=2 M_{\text {mono }} \times \sqrt{1-\frac{\omega^{2}}{m^{2}}}<2 M_{\text {mono }}
$$

The mass of the mesonic bound state is smaller than the sum of the masses of isolated monopole and anti-monopole.

We show how the magnetic meson varies in one period $T=\frac{2 \pi}{\omega}$ in figure 7 . The sources of outgoing magnetic field are identified as slender monopoles and those of incoming magnetic field as slender anti-monopoles. It is interesting to observe that the meson is made of $\mathcal{M}_{0}$ and $\overline{\mathcal{M}}_{0}$ at an instance (for example $t=T / 4$ ), and that it transforms into a different meson made of $\mathcal{M}_{\pi}$ and $\overline{\mathcal{M}}_{\pi}$ at another instance (for example $t=3 T / 4$ ). In figure 7 , we also show the topological charge density $\mathcal{Q}_{\mathrm{m}}$ given in eq. (5.27) together with the energy density of the electric field

$$
\mathcal{E}=\frac{1}{g^{2}} \operatorname{Tr}\left[\left(F_{01}\right)^{2}+\left(F_{02}\right)^{2}\right]=\frac{1}{g^{2}}|1-z \partial \psi|^{2} e^{-\psi}\left(\partial_{t} \tilde{\Theta}\right)^{2} .
$$


As the monopole and anti-monopole approach each other, the magnetic energy density $\mathcal{M}$ decreases and the electric field energy density $\mathcal{E}$ grows. At the very instance of collision, the magnetic energy disappears and is transferred into the electric energy completely. The electric field is generated by the time variation (decrease) of the magnetic field as monopole and anti-monopole merge.

\subsection{Scattering of the slender monopole and anti-monopole}

Let us next study the head-on collision of the slender monopole and anti-monopole. There are two types of collisions: one type is the collision of $\mathcal{M}_{0}$ and $\overline{\mathcal{M}}_{0},\left(\mathcal{M}_{\pi}\right.$ and $\left.\overline{\mathcal{M}}_{\pi}\right)$ and the other type is that of $\mathcal{M}_{0}$ and $\overline{\mathcal{M}}_{\pi}\left(\mathcal{M}_{\pi}\right.$ and $\left.\overline{\mathcal{M}}_{0}\right)$.

Scattering of $\mathcal{M}_{\mathbf{0}}$ and $\overline{\mathcal{M}}_{\mathbf{0}}\left(\mathcal{M}_{\boldsymbol{\pi}}\right.$ and $\left.\overline{\mathcal{M}}_{\boldsymbol{\pi}}\right)$. The exact solution for the moduli field for the collision of a monopole and anti-monopole of the same species $\left(\mathcal{M}_{0}\right.$ or $\left.\mathcal{M}_{\pi}\right)$ is given by

$$
\tilde{\Theta}=2 \arctan \left(\frac{\sinh m u \gamma x^{0}}{u \cosh m \gamma x^{3}}\right), \quad \gamma=\frac{1}{\sqrt{1-u^{2}}} .
$$

The parameter $u$ corresponds to the velocity of the monopole. However, we should keep in mind that our approximation holds only for small velocities, that is

$$
u \ll 1 \quad(\gamma \simeq 1) .
$$

Since we are using the rigid-body approximation we cannot faithfully describe Lorentz boosted monopoles. Thus even though we can solve the 1+1-dimensional effective dynamics for arbitrary velocities, the full 1+3-dimensional dynamics is correctly represented only within the restriction of eq. (5.42). A typical configuration is shown in figure 8. The slender magnetic monopole $\mathcal{M}_{\pi}$ comes from the left infinity and the anti-monopole $\overline{\mathcal{M}}_{\pi}$ comes from the right infinity. As they approach to the collision point, the magnetic energy decreases while the electric energy grows. After the collision, the magnetic energy grows as the monopole $\mathcal{M}_{0}$ (anti-monopole $\overline{\mathcal{M}}_{0}$ ) goes toward the left (right) infinity. Thus we find that the species of the monopole and the anti-monopole changes after the collision. The attractive force given in eq. (5.36) gives rise to a negative time delay

$$
\delta t=\frac{1-u^{2}}{u} \log u<0 .
$$

Scattering of $\mathcal{M}_{0}$ and $\overline{\mathcal{M}}_{\boldsymbol{\pi}}$ (or $\mathcal{M}_{\boldsymbol{\pi}}$ and $\left.\overline{\mathcal{M}}_{\mathbf{0}}\right)$. The solution for the scattering of a monopole and an anti-monopole of different species is given by

$$
\tilde{\Theta}=2 \arctan \left(\frac{u \sinh m \gamma x^{3}}{\cosh m u \gamma x^{0}}\right) \text {. }
$$

A typical configuration is shown in figure 9 . In contrast to the previous type of scattering the species of monopoles $\left(\mathcal{M}_{0}\right.$ or $\left.\mathcal{M}_{\pi}\right)$ do not change into different species during the collision. As shown in figure 9 , the anti-monopole $\overline{\mathcal{M}}_{\pi}$ comes from the left infinity and reflects back toward the left infinity, while the monopole $\mathcal{M}_{0}$ comes from the right infinity and reflects back toward the right infinity. 

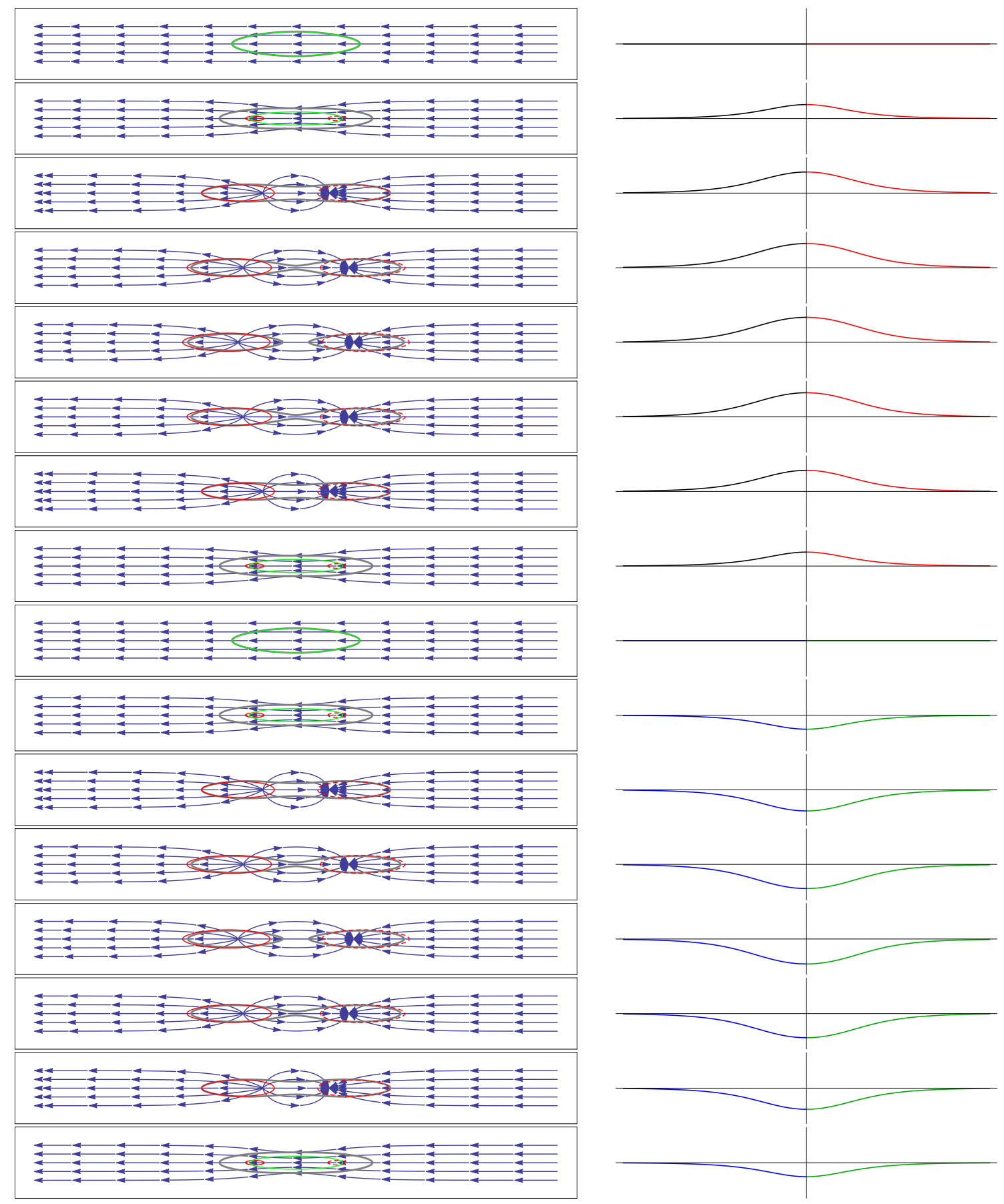

Figure 7. Snapshots of a single period of the magnetic meson. The top is at $t=0$ and the bottom is at $t=T-\delta t$ with $\delta t=T / 16$. The left panel shows the magnetic field $\left(F_{12}^{\Sigma}, F_{23}^{\Sigma}\right)$ by blue streamlines and the topological charge densities, $\mathcal{M}= \pm 0.017$, electric energy density, $\mathcal{E}=0.012$, and the dressed energy density $\mathcal{H}_{\text {dress }}=0.02$ by red/green/grey contours. In the right figures, $\tilde{\Theta}\left(x^{3}, t\right)$ is plotted. The curves are piecewise colored by black, red, blue and green for $\mathcal{M}_{0}, \overline{\mathcal{M}}_{0}$, $\mathcal{M}_{\pi}$ and $\overline{\mathcal{M}}_{\pi}$, respectively. We set $g v=1, m=1 / 5$ and $\omega=1 / 10 . x^{1} \in[-3,3]$ and $x^{3} \in[-30,30]$. 

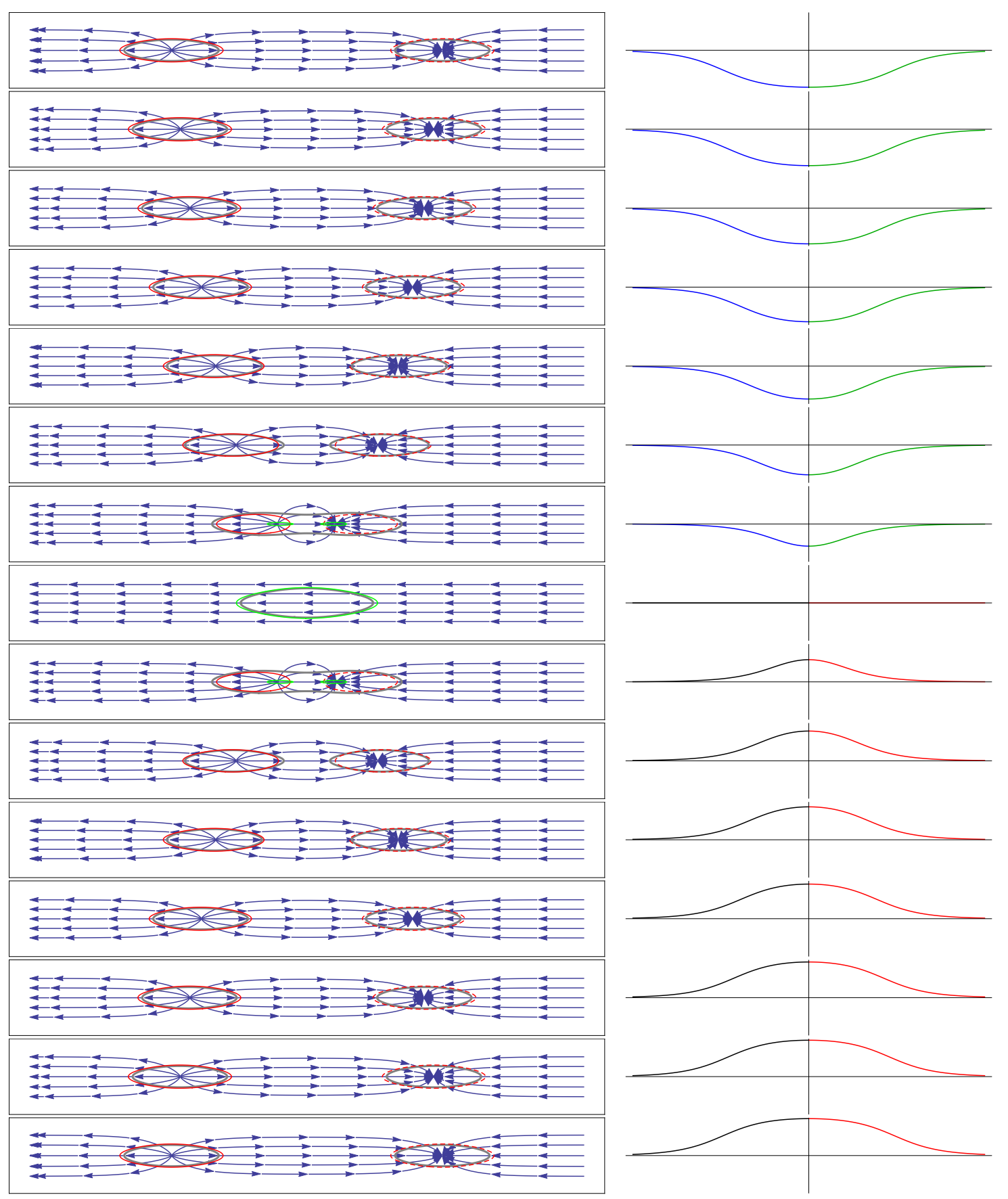

Figure 8. Snapshots (from $t=-42$ to $t=42$ with $\delta t=6$ interval) of scattering of the slender monopole and anti-monopole. The red/green/gray contours are $\left(\mathcal{M}, \mathcal{E}, \mathcal{H}_{\text {dress }}\right)=$ $( \pm 0.017,0.01,0.02)$, see the caption of figure 7 for explanation. We set $g v=1, m=1 / 5$ and $u=1 / 10 . x^{1} \in[-3,3]$ and $x^{3} \in[-30,30]$. 

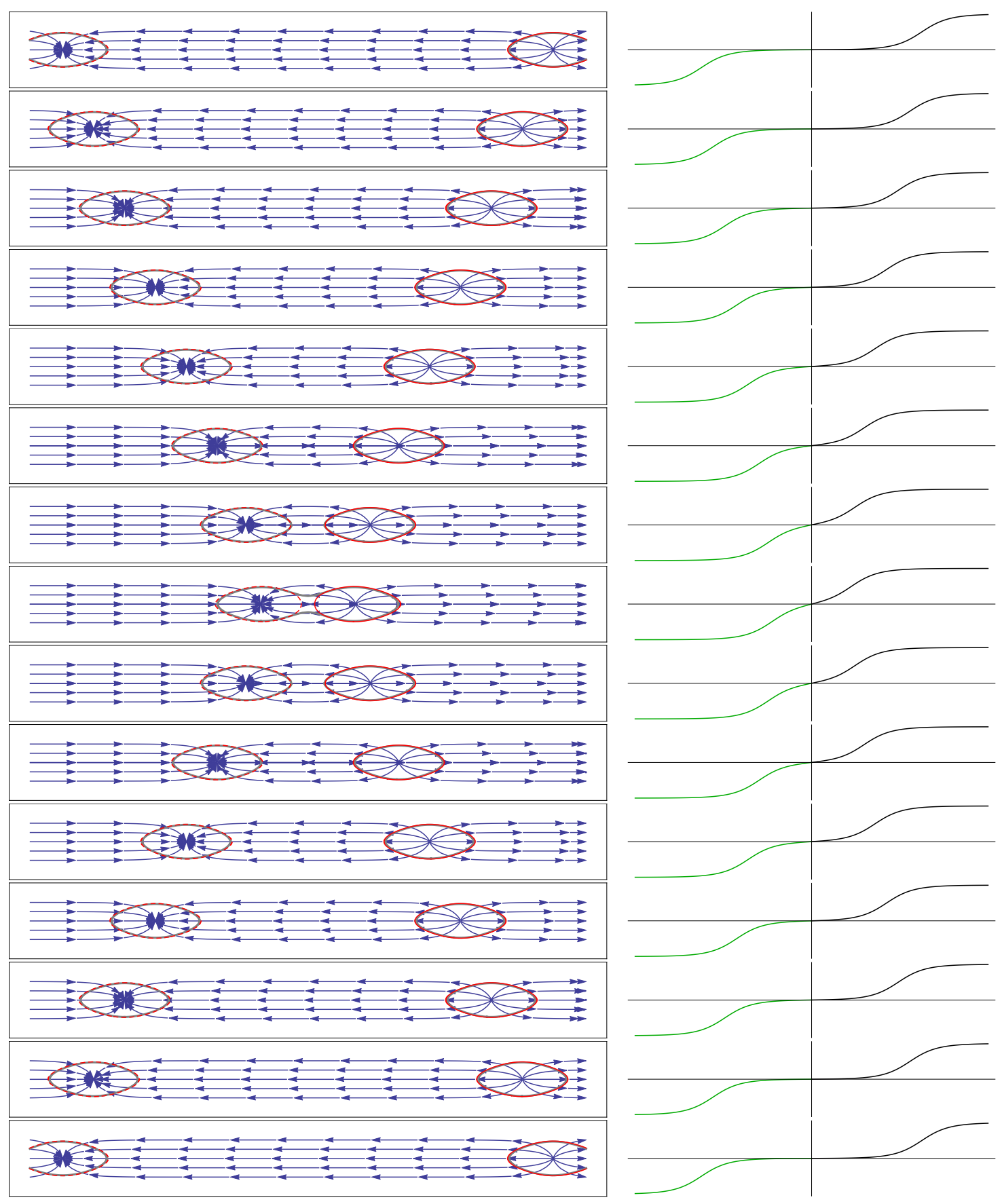

Figure 9. Snapshots (from $t=-70$ to $t=70$ with $\delta t=10$ interval) of scattering of the slender monopole and anti-monopole. The red/gray contours are $\left(\mathcal{M}, \mathcal{H}_{\text {dress }}\right)=( \pm 0.017,0.02)$. See the caption of figure 7 for explanation. We set $g v=1, m=1 / 3$ and $u=1 / 3 . \quad x^{1} \in[-3,3]$ and $x^{3} \in[-30,30]$. 


\subsection{Lifting the zero mode}

So far, we have considered only solutions with a constant azimuthal angle $\tilde{\Phi}=$ const., where all the solutions are mapped onto trajectories on a great circle of $\mathbb{C} P^{1}$. Since the fundamental homotopy group of the total target space $\mathbb{C} P^{1} \simeq S^{2}$ of the $\mathrm{U}(2)$ vortex is trivial, a small fluctuation of $\tilde{\Phi}$ may destabilize our solutions with constant $\tilde{\Phi}$ and can convert the magnetic mesons from bound states (of monopole and anti-monopole) into resonances. This observation appears plausible, but we would like to emphasize that our configurations with constant $\tilde{\Phi}$ are all solutions of the full equations of motion (within our approximation). Therefore, our statement that the monopole and anti-monopole do not always decay into radiation but can make bound states or resonances is still valid. Moreover, one should note that these magnetic mesons play a significant role in understanding dynamics of monopole and anti-monopole system, irrespective of whether they are genuine bound states or resonances.

If one desires, one can introduce a small scalar potential such as

$$
V_{\text {add }}=\lambda g^{2} m^{2}\left(\operatorname{Tr}\left[H \sigma_{1} H^{\dagger}\right]\right)^{2} \text {. }
$$

This potential respects the gauge symmetry $\mathrm{U}(2)_{\mathrm{C}}$ while the flavor symmetry $\mathrm{U}(1)_{\mathrm{F} 3}$ is no longer genuine symmetry of the system. It is the approximate symmetry, and the zero mode of the azimuthal angle $\tilde{\Phi}$ is weakly lifted. Let us see how this additional potential gives a finite mass to $\tilde{\Phi}$. First of all, we assume that $V_{\text {add }}$ is sufficiently small in such a way that the rigid-body approximation works. Namely, the zero-th order equations (4.11) and (4.12), and the first order equations (4.21) and (4.22) are intact. Thus, to the first order of the expansion in $\epsilon$, solutions are the same as those for no additional potential given in eqs. (4.20), (4.23), and (4.24). On the other hand, the quasi zero mode $\phi\left(x^{0}, x^{3}\right)$ may receive a correction from $V_{\text {add }}$. Plugging the zero-th order configuration $H^{(0)}\left(x^{1}, x^{2} ; \phi\left(x^{0}, x^{3}\right)\right)$ into eq. (5.45), we obtain the following expression

$$
V_{\text {add }}=\frac{\lambda m^{2}}{g^{2}} F^{2} \times\left(\frac{\phi+\bar{\phi}}{1+|\phi|^{2}}\right)^{2}
$$

where $F$ is defined in eq. (4.27). This potential should be think of as a correction of order $\epsilon^{2}$ for the quasi zero mode. Indeed, it is possible if we choose $\lambda$ to be a parameter of $\mathcal{O}(1)$. After integrating in $x^{1}$ and $x^{2}$, one obtains

$$
\mathcal{L}_{\text {eff }+ \text { add }}=\frac{4 \pi}{g^{2}} \frac{\left|\partial_{\alpha} \phi\right|^{2}-m^{2}|\phi|^{2}-\tilde{m}^{2}(\phi+\bar{\phi})^{2}}{\left(1+|\phi|^{2}\right)^{2}}
$$

with

$$
\tilde{m}^{2}=m^{2} \times \frac{\lambda}{4 \pi} \int F^{2} d^{2} x \sim \mathcal{O}\left(m^{2}\right) .
$$

All the terms here are of order $\epsilon^{2}$ compared to the leading term $2 \pi v^{2}$, as we desired. In this way, the new mass $\tilde{m}$ appears, which sets the phase of $\phi$ zero or $\pi$. In summary, the stability of the solution with $\tilde{\Phi}=0$ is assured by introducing $V_{\text {add }}$. 


\section{Dyon in the Higgs phase}

Another non-trivial application of the rigid-body approximation is a dyonic solution in the non-Abelian superconductor. The corresponding solution in $3+1$ dimensions are known as 1/4 BPS state [51-53]. A time-dependent stationary solution of the equations of motion (4.32) and (4.33) is given by

$$
\Theta\left(x^{0}, x^{3}\right)=2 \arctan \left(e^{\sqrt{m^{2}-\omega^{2}} x^{3}}\right), \quad \Phi\left(x^{0}, x^{3}\right)=\omega x^{0},
$$

with $\omega<m$. Here, we again use the coordinates $\Theta$ and $\Phi$. This is called a Q-kink solution [46, 47], which carries both topological (magnetic) and Noether (electric) charges. Let us see how the above solution can be derived through a standard Bogomol'nyi technique. The $1+1$ dimensional Hamiltonian corresponding to the effective Lagrangian (4.31) can be cast into the following perfect square form as

$$
\begin{aligned}
\mathcal{H}_{\text {eff }}= & \frac{\pi}{g^{2}}\left[\dot{\Theta}^{2}+\Theta^{\prime 2}+\sin ^{2} \Theta\left(\dot{\Phi}^{2}+\Phi^{\prime 2}\right)+m^{2} \sin ^{2} \Theta\right] \\
= & \frac{\pi}{g^{2}}\left[\dot{\Theta}^{2}+\sin ^{2} \Theta \Phi^{\prime 2}+\left(\Theta^{\prime}-m \cos \alpha \sin \Theta\right)^{2}+\sin ^{2} \Theta(\dot{\Phi}-m \sin \alpha)^{2}\right. \\
& \left.\quad+2 m \Theta^{\prime} \cos \alpha \sin \Theta+2 m \dot{\Phi} \sin \alpha \sin ^{2} \Theta\right] \\
\geq & \frac{2 \pi m}{g^{2}}\left(\Theta^{\prime} \cos \alpha \sin \Theta+\dot{\Phi} \sin \alpha \sin ^{2} \Theta\right) .
\end{aligned}
$$

Here $\alpha$ is an arbitrary constant. Integrating this over the $x^{3}$ direction, one obtains the following inequality

$$
\int d x^{3} \mathcal{H}_{\mathrm{eff}} \geq \frac{4 \pi m}{g^{2}}(T \cos \alpha+N \sin \alpha)
$$

where we defined the topological charge and the Noether charge by

$$
\begin{aligned}
T & =\int d x^{3} \frac{1}{2} \Theta^{\prime} \sin \Theta=-\frac{1}{2}[\cos \Theta]_{x^{3} \rightarrow-\infty}^{x^{3} \rightarrow \infty}, \\
N & =\int d x^{3} \frac{1}{2} \dot{\Phi} \sin ^{2} \Theta .
\end{aligned}
$$

Since the parameter $\alpha$ is arbitrary, the strictest bound for given $T$ and $N$ is obtained when it holds

$$
\tan \alpha=\frac{N}{T}
$$

The bound is saturated for solutions for the following first order equations

$$
\Theta^{\prime}=\frac{m T}{\sqrt{T^{2}+N^{2}}} \sin \Theta, \quad \dot{\Phi}=\frac{m N}{\sqrt{T^{2}+N^{2}}},
$$

with the energy

$$
\int x^{3} \mathcal{H}_{\mathrm{eff}}=\frac{4 \pi m}{g^{2}} \sqrt{T^{2}+N^{2}} .
$$

The solution given in eq. (6.1) corresponds to the solution with the charges

$$
T=1, \quad N=\frac{\omega}{\sqrt{m^{2}-\omega^{2}}}
$$


Thus, the energy is given by

$$
\int d x^{3} \mathcal{H}_{\mathrm{eff}}=\frac{4 \pi m}{g^{2}} \frac{m}{\sqrt{m^{2}-\omega^{2}}}
$$

Let us see this configuration in $1+1$ dimensions from the $3+1$ dimensional perspective. The corresponding electric and magnetic fields can be easily obtained from $\bar{A}^{(0)}$ in eq. (4.38) and $A_{\alpha}^{(1)}$ in eq. (4.23) by noting that $x^{0}, x^{3}$ dependence resides only in $\Theta$ and $\Phi$ in eq. (6.1) which appears through $U\left(x^{0}, x^{3}\right)$ in eqs. (4.18), (4.30). The Abelian electric and magnetic fields are given as

$$
F_{12}^{0} \simeq-\partial \bar{\partial} \psi, \quad F_{23}^{0} \simeq 0, \quad F_{31}^{0} \simeq 0, \quad F_{01}^{0}=F_{02}^{0}=F_{03}^{0}=0,
$$

and their non-Abelian counterparts as

$$
\begin{aligned}
& B_{3}^{\Sigma}=F_{12}^{\Sigma} \simeq \partial \bar{\partial} \psi \tanh \left(\sqrt{m^{2}-\omega^{2}} x^{3}\right), \\
& B_{1}^{\Sigma}=F_{23}^{\Sigma} \simeq \frac{\sqrt{m^{2}-\omega^{2}}}{4} \partial_{1}\left(r^{2} e^{-\psi}\right) \operatorname{sech}^{2}\left(\sqrt{m^{2}-\omega^{2}} x^{3}\right), \\
& B_{2}^{\Sigma}=F_{31}^{\Sigma} \simeq \frac{\sqrt{m^{2}-\omega^{2}}}{4} \partial_{2}\left(r^{2} e^{-\psi}\right) \operatorname{sech}^{2}\left(\sqrt{m^{2}-\omega^{2}} x^{3}\right), \\
& E_{1}^{\Sigma}=F_{01}^{\Sigma} \simeq \frac{\omega}{4} \partial_{1}\left(r^{2} e^{-\psi}\right) \operatorname{sech}^{2}\left(\sqrt{m^{2}-\omega^{2}} x^{3}\right), \\
& E_{2}^{\Sigma}=F_{02}^{\Sigma} \simeq \frac{\omega}{4} \partial_{2}\left(r^{2} e^{-\psi}\right) \operatorname{sech}^{2}\left(\sqrt{m^{2}-\omega^{2}} x^{3}\right), \\
& E_{3}^{\Sigma}=F_{03}^{\Sigma} \simeq 0 .
\end{aligned}
$$

$F_{03}$ is of order $\mathcal{O}\left(\epsilon^{2}\right)$ by definition. Then we can define topological and electric charge densities as

$$
\begin{aligned}
\mathcal{Q}_{\mathrm{m}} & =\frac{1}{g} \operatorname{div} \vec{B}^{\Sigma}=\frac{1}{g} \sqrt{m^{2}-\omega^{2}} \operatorname{sech}^{2}\left(\sqrt{m^{2}-\omega^{2}} x^{3}\right)\left(\partial \bar{\partial}-\frac{4}{g^{2} v^{2}}(\partial \bar{\partial})^{2}\right) \psi, \\
\mathcal{Q}_{\mathrm{e}} & =\frac{1}{g} \operatorname{div} \vec{E}^{\Sigma}=\frac{1}{g} \omega \operatorname{sech}^{2}\left(\sqrt{m^{2}-\omega^{2}} x^{3}\right)\left(-\frac{4}{g^{2} v^{2}}(\partial \bar{\partial})^{2} \psi\right) .
\end{aligned}
$$

Note that the factor $\omega \operatorname{sech}^{2}\left(\sqrt{m^{2}-\omega^{2}} x^{3}\right)$ appearing in $\mathcal{Q}_{\mathrm{e}}$ corresponds to the integrand of the Noether charge $N$ of $Q$-kink, eq. (6.5), as

$$
\dot{\Phi} \sin ^{2} \Theta=\omega \operatorname{sech}^{2}\left(\sqrt{m^{2}-\omega^{2}} x^{3}\right)
$$

In this way, one realizes that the electric charge density $\mathcal{Q}_{\mathrm{e}}$ is a direct manifestation of the Noether charge density of the $Q$-kink, as should be expected. Since the magnetic and electric fields except for $F_{12}^{\Sigma}$ go to zero at spatial infinity, total topological and electric charges are given by

$$
Q_{\mathrm{m}}=\frac{2 \pi}{g}, \quad Q_{\mathrm{e}}=0
$$

Although the total electric charge is zero, its density is not equal to zero in contrast to the case of the monopole and anti-monopole scattering, see eq. (5.30). Similarly to a neutron, there is a non-trivial charge density distribution inside the monopole. The electric and magnetic charge densities are shown in figure 10. 

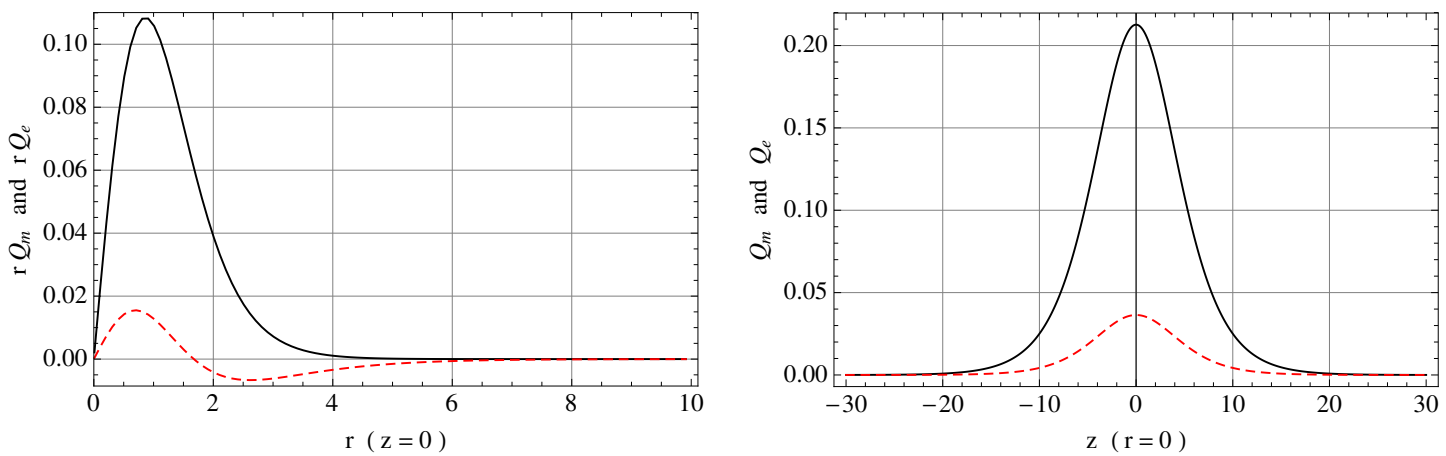

Figure 10. The electric and magnetic charge distribution of a slender dyonic monopole in the Higgs phase. The left panel shows the magnetic (black solid line) $r \mathcal{Q}_{\mathrm{m}}$ and electric (red dashed line) $r \mathcal{Q}_{\mathrm{e}}$ charge densities multiplied by $r$ at $z=0$ as a function of $r$. The right panel shows those at $r=0$ as a function of $z$. The parameters are set as $g v=1, m=1 / 5$ and $\omega=1 / 10$.

Let us compare the dyonic monopoles in the Coulomb phase and the Higgs phase. In the Coulomb phase, the conserved Noether charge of the unbroken U(1) gauge symmetry is associated with the time dependent internal moduli $S^{1}$ of the static monopole. Since there is no fields which would screen the electric charge, the total electric charge can be non zero in the Coulomb phase [54]. On the other hand, the U(1) symmetry is broken in the Higgs phase, but it is locked with the flavor symmetry of the fundamental Higgs field $H$. Therefore, the non-zero Noether charge of flavor U(1) symmetry also exists. However, since $H$ is charged under the $\mathrm{U}(1)$ symmetry and it is condensed in the Higgs phase, the electric charge of the monopole generated by the rotation of the $\mathrm{U}(1)$ phase is screened. Thus, the total electric charge becomes zero in the Higgs phase.

By flipping the sign of $\omega$, one can also construct the slender dyonic monopole with opposite electric charge density distribution. It is also easy to construct dyonic antimonopoles by flipping the topological charge.

\section{Conclusion and discussion}

In this paper we have investigated the low energy dynamics of monopoles and antimonopoles in the non-Abelian superconductor. We have restricted ourselves to the parameter region $m \ll g v$ where the monopoles are of slender ellipsoidal shape, confined on a vortex string, with the cross-section comparable to that of the monopole. For that reason, the scattering problem becomes essentially $1+1$ dimensional. Indeed, we have found that at least a part of the low energy dynamics is identical to the sine-Gordon system in $1+1$ dimensions up to the first order of the expansion in $\epsilon=m /(g v)$, when $\left\{m, \partial_{0}, \partial_{3}\right\} \ll\left\{g v, \partial_{1}, \partial_{2}\right\}$ holds. This observation is very useful because the sine-Gordon system is solvable. In the literature, only the static kink was identified with the monopole. In this paper, we have dealt with all the sine-Gordon solutions and have constructed the dictionary with which one can easily translate the dynamics of sine-Gordon kinks in $1+1$ dimensions into the dynamics of monopoles in $1+3$ dimensions. A surprising fact is that the monopole and anti-monopole do not always decay into radiation when they make a head- 
on collision, although they are not protected by topology. We have studied three concrete examples: (1) the magnetic meson which is the bound state of the slender monopole and anti-monopole, (2) the scattering of the monopole and anti-monopole of the same species, and (3) the scattering of the monopole and anti-monopole of the different species. All these three examples show that the monopole and anti-monopole do not always annihilate. This observation may be counter-intuitive and remarkable.

In order to illustrate the usefulness of our dictionary, let us also give a solution of three body scattering. Three body system is in general very complicated. However, due to the power of integrability, we can easily describe the three body collision of a magnetic meson and a monopole:

$$
\begin{aligned}
& \tilde{\Theta}=2 \tan ^{-1} e^{\theta_{2}}+2 \tan ^{-1}\left[\frac { a _ { 1 } + a _ { 3 } } { a _ { 1 } - a _ { 3 } } \operatorname { t a n } \left\{\tan ^{-1} \frac{\left(a_{1}+a_{2}\right) \sinh \left(\theta_{1}-\theta_{2}\right) / 2}{\left(a_{1}-a_{2}\right) \cosh \left(\theta_{1}+\theta_{2}\right) / 2}\right.\right. \\
& \theta_{n}=\frac{a_{n}^{2}+1}{2 a_{n}}\left(m x^{3}+\frac{a_{n}^{2}-1}{a_{n}^{2}+1} m x^{0}\right) \quad(n=1,2,3), \\
& a_{1}=\sqrt{\frac{1-b}{1+b}}\left(\sqrt{\left.1-a_{2}+a_{3}\right) \sinh \left(\theta_{2}-\theta_{3}\right) / 2}-i \omega\right), \\
& a_{2}=\sqrt{\frac{1-k}{1+k}}, \\
& a_{3}=\sqrt{\frac{1-b}{1+b}}\left(\sqrt{\left.1-\theta_{2}+\theta_{3}\right) / 2}+i \omega\right) .
\end{aligned}
$$

Here $b$ is the velocity of the magnetic meson, $k$ is that of the isolated monopole, and $\omega$ is the frequency of the magnetic meson. The solution is shown in figure 11. Of course, this is an example and one can easily add any number of monopoles and anti-monopoles by using well established methods, such as the Bäcklund transformation.

Let us discuss a number of points about our results and future directions of our research in the following.

(I) We have studied the specific parameter region $m \ll g v$. We have made this choice in order to utilize the rigid-body approximation, which leads to a nice mapping of the $1+3$ dimensional problem into the integrable sine-Gordon system in $1+1$ dimensions. However, we can study only the low energy dynamics with our approximation. In order to go beyond the approximation, we need either to include higher order corrections or to solve the full equations of motion. Although solving $1+3$ dimensional second order differential equations is in general not an easy task, it is worth doing for the following reasons. Firstly, it is a direct check of the validity of our approximation. Secondly, the monopoles in the parameter region $m \gg g v$ are close to spherical, and their dynamics can be very different from those of the slender monopoles studied in this work. Numerical works may be needed, since there is little chance to solve the full equations of motion in the whole range of parameter space by analytic methods. However, so far, no numerical solution even for the static single monopole in the nonAbelian superconductor has been constructed (only approximate analytical solution is known [40]). We hope to do the numerical works and to report the results elsewhere. 

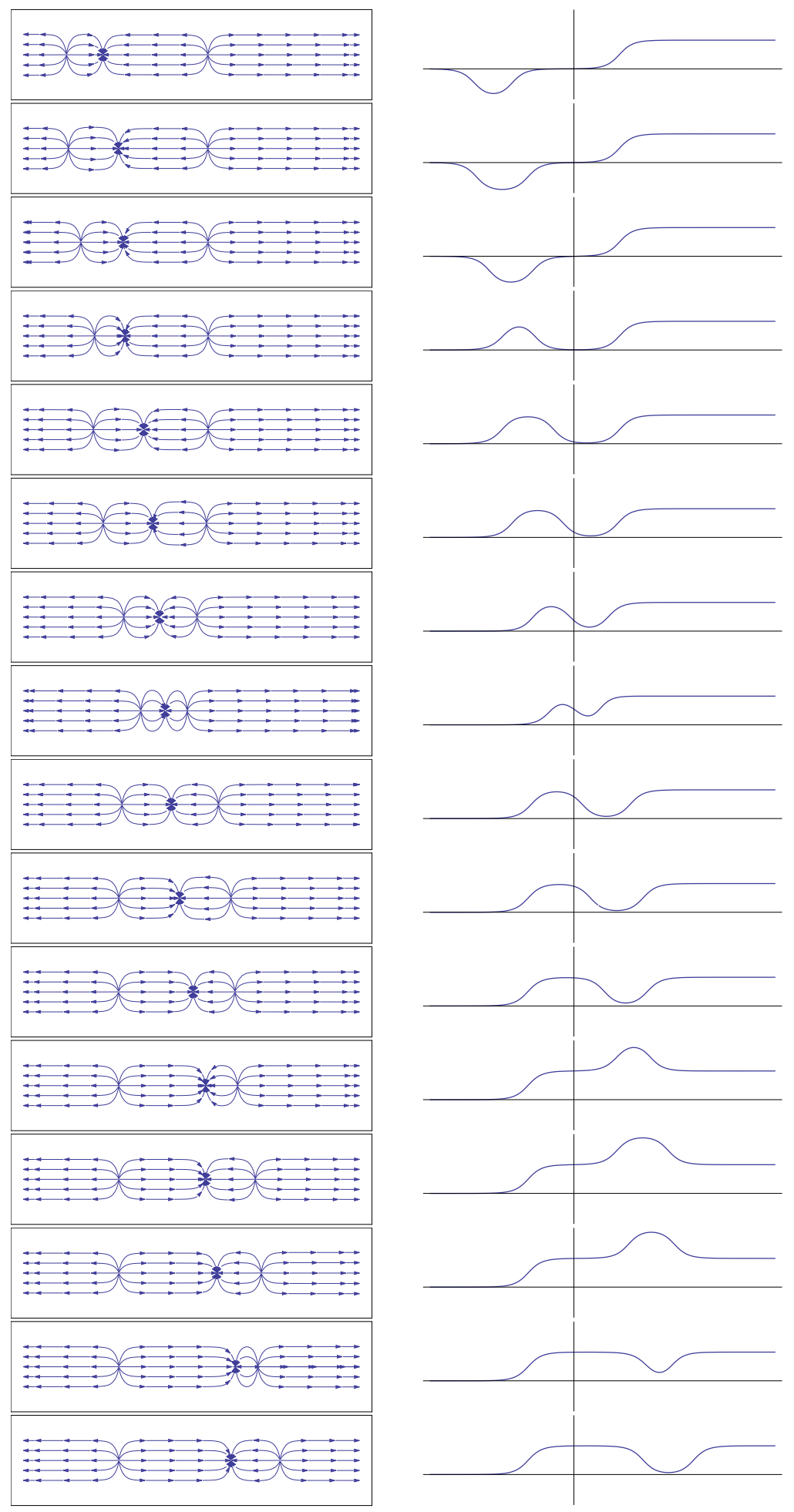

Figure 11. The scattering of the magnetic meson and the isolated monopole. We set $g v=1$, $m=1 / 3$ and $\omega=1 / 10, b=1 / 10, k=0, x^{1} \in[-2.5,2.5], x^{3} \in[-50,70]$. The snapshots are taken for $x^{0} \in[-200,250]$ with $\delta t=30$ interval. 
(II) We have studied the scattering of the slender monopole and anti-monopole but have not studied the dynamics of two monopoles. This is because the monopoles are put in the non-Abelian superconductor. Due to the flux conservation, two identical monopoles are not allowed to be next to each other on a vortex-string. Either an anti-monopole should be sandwiched between them or the monopoles should sit on different vortex strings. The former type of configuration is precisely those studied in this work. The latter configuration gives almost decoupled monopoles and are uninteresting. On the other hand, the gauge theory with higher rank gauge group such as $\mathrm{U}(N)$ with $N \geq 3$ provides a new possibility: there are several species of monopoles which can be put next to each other on a vortex string. We leave an interesting dynamics of such monopoles for future investigations.

(III) In this work, we have studied the monopole and vortex string in the U(2) gauge theory, where only Abelian vortices and Abelian monopoles are possible. NonAbelian vortex is defined as a vortex with non-Abelian orientational moduli, and non-Abelian monopoles are those that can interact with such non-Abelian vortices. These non-Abelian vortices and non-Abelian monopoles can arise, if higher rank gauge theories such as $\mathrm{U}(3)$ are considered. In general, dynamics of Abelian solitons and non-Abelian solitons are quite different. For instance, non-Abelian domain walls have been studied in comparison to Abelian domain walls in ref. [49]. Moreover, the non-Abelian vortex and non-Abelian monopole are believed to be very relevant to the question of confinemnt. The dynamics of non-Abelian monopoles in the non-Abelian superconductor has not been examined, but it is worthwhile to study it in detail. We also leave this problem as a future work.

(IV) The solutions which we considered in this work are all noncompact because they are accompanied by the infinitely long vortex string. It is also interesting to study the dynamics of monopoles on a curved vortex string. Especially, one may consider a vortex ring (which is called a vorton) [50]. It is interesting to pursue the similarity between bound states of topological solitons and bound states (mesons) of elementary constituents (quarks).

\section{Acknowledgments}

M.A. and F.B. is supported by the Research Program MSM6840770029 and by the project of International Cooperation ATLAS-CERN of the Ministry of Education, Youth and Sports of the Czech Republic. This work is supported by Grant-in Aid for Scientific Research No.25400280 (M. A. ), No.23740226 and No.26800119 (M. E. ), and No. 25400241 (N. S. ) from the Ministry of Education, Culture, Sports, Science and Technology (MEXT) of Japan.

Open Access. This article is distributed under the terms of the Creative Commons Attribution License (CC-BY 4.0), which permits any use, distribution and reproduction in any medium, provided the original author(s) and source are credited. 


\section{References}

[1] N.S. Manton and P. Sutcliffe, Topological solitons, Cambridge Univ. Pr., Cambridge U.K. (2004) [INSPIRE].

[2] D. Tong, TASI lectures on solitons: instantons, monopoles, vortices and kinks, hep-th/0509216 [INSPIRE].

[3] M. Eto, Y. Isozumi, M. Nitta, K. Ohashi and N. Sakai, Solitons in the Higgs phase: the moduli matrix approach, J. Phys. A 39 (2006) R315 [hep-th/0602170] [INSPIRE].

[4] M. Shifman and A. Yung, Supersymmetric solitons and how they help us understand non-Abelian gauge theories, Rev. Mod. Phys. 79 (2007) 1139 [hep-th/0703267] [INSPIRE].

[5] P.A.M. Dirac, Quantized singularities in the electromagnetic field, Proc. Roy. Soc. Lond. A 133 (1931) 60 [INSPIRE].

[6] Y. Nambu, Strings, monopoles and gauge fields, Phys. Rev. D 10 (1974) 4262 [INSPIRE].

[7] S. Mandelstam, Vortices and quark confinement in non-Abelian gauge theories, Phys. Lett. B 53 (1975) 476 [INSPIRE].

[8] S. Mandelstam, Vortices and quark confinement in non-Abelian gauge theories, Phys. Rept. 23 (1976) 245 [INSPIRE].

[9] G. 't Hooft, Topology of the gauge condition and new confinement phases in non-Abelian gauge theories, Nucl. Phys. B 190 (1981) 455 [inSPIRE].

[10] N. Seiberg and E. Witten, Electric-magnetic duality, monopole condensation and confinement in $N=2$ supersymmetric Yang-Mills theory, Nucl. Phys. B 426 (1994) 19 [Erratum ibid. B 430 (1994) 485] [hep-th/9407087] [INSPIRE].

[11] M.G. Alford, K. Rajagopal and F. Wilczek, QCD at finite baryon density: nucleon droplets and color superconductivity, Phys. Lett. B 422 (1998) 247 [hep-ph/9711395] [INSPIRE].

[12] M.G. Alford, K. Rajagopal and F. Wilczek, Color flavor locking and chiral symmetry breaking in high density QCD, Nucl. Phys. B 537 (1999) 443 [hep-ph/9804403] [InSPIRE].

[13] R. Rapp, T. Schäfer, E.V. Shuryak and M. Velkovsky, Diquark Bose condensates in high density matter and instantons, Phys. Rev. Lett. 81 (1998) 53 [hep-ph/9711396] [INSPIRE].

[14] A.P. Balachandran, S. Digal and T. Matsuura, Semi-superfluid strings in high density QCD, Phys. Rev. D 73 (2006) 074009 [hep-ph/0509276] [InSPIRE].

[15] E. Nakano, M. Nitta and T. Matsuura, Non-Abelian strings in high density QCD: zero modes and interactions, Phys. Rev. D 78 (2008) 045002 [arXiv:0708.4096] [INSPIRE].

[16] A. Hanany and D. Tong, Vortices, instantons and branes, JHEP 07 (2003) 037 [hep-th/0306150] [INSPIRE].

[17] A. Hanany and D. Tong, Vortex strings and four-dimensional gauge dynamics, JHEP 04 (2004) 066 [hep-th/0403158] [INSPIRE].

[18] R. Auzzi, S. Bolognesi, J. Evslin, K. Konishi and A. Yung, Non-Abelian superconductors: vortices and confinement in $N=2$ SQCD, Nucl. Phys. B 673 (2003) 187 [hep-th/0307287] [INSPIRE].

[19] M. Shifman and A. Yung, Non-Abelian string junctions as confined monopoles, Phys. Rev. D 70 (2004) 045004 [hep-th/0403149] [INSPIRE]. 
[20] A. Gorsky, M. Shifman and A. Yung, Confined magnetic monopoles in dense QCD, Phys. Rev. D 83 (2011) 085027 [arXiv:1101.1120] [INSPIRE].

[21] M. Eto, M. Nitta and N. Yamamoto, Confined monopoles induced by quantum effects in dense QCD, Phys. Rev. D 83 (2011) 085005 [arXiv:1101.2574] [InSPIRE].

[22] M. Eto, Y. Hirono, M. Nitta and S. Yasui, Vortices and other topological solitons in dense quark matter, Prog. Theor. Exp. Phys. 2014 (2014) 012D01 [arXiv:1308.1535] [InSPIRE].

[23] E.B. Bogomolny, Stability of classical solutions, Sov. J. Nucl. Phys. 24 (1976) 449 [Yad. Fiz. 24 (1976) 861] [INSPIRE].

[24] M.K. Prasad and C.M. Sommerfield, An exact classical solution for the 't Hooft monopole and the Julia-Zee dyon, Phys. Rev. Lett. 35 (1975) 760 [InSPIRE].

[25] E. Witten and D.I. Olive, Supersymmetry algebras that include topological charges, Phys. Lett. B 78 (1978) 97 [INSPIRE].

[26] G. 't Hooft, Magnetic monopoles in unified gauge theories, Nucl. Phys. B 79 (1974) 276 [INSPIRE].

[27] A.M. Polyakov, Particle spectrum in the quantum field theory, JETP Lett. 20 (1974) 194 [Pisma Zh. Eksp. Teor. Fiz. 20 (1974) 430] [InSPIRE].

[28] N.S. Manton, A remark on the scattering of BPS monopoles, Phys. Lett. B 110 (1982) 54 [INSPIRE].

[29] G.W. Gibbons and N.S. Manton, The moduli space metric for well separated BPS monopoles, Phys. Lett. B 356 (1995) 32 [hep-th/9506052] [INSPIRE].

[30] M.F. Atiyah and N.J. Hitchin, The geometry and dynamics of magnetic monopoles. M.B. Porter lectures, Princeton Univ. Pr., Princeton U.S.A. (1988) [INSPIRE].

[31] C.H. Taubes, The existence of a nonminimal solution to the SU(2) Yang-Mills Higgs equations on $R^{3}$, Commun. Math. Phys. 86 (1982) 257 [inSPIRE].

[32] M. Oleszczuk and E. Werner, Monopole-anti-monopole pair solution of the classical SU(3) Yang-Mills theory, Phys. Rev. D 35 (1987) 3225 [inSPIRE].

[33] T.A. Ioannidou and P.M. Sutcliffe, Non-Bogomolny SU(N) BPS monopoles, Phys. Rev. D 60 (1999) 105009 [hep-th/9905169] [INSPIRE].

[34] B. Kleihaus and J. Kunz, A monopole-anti-monopole solution of the SU(2) Yang-Mills Higgs model, Phys. Rev. D 61 (2000) 025003 [hep-th/9909037] [INSPIRE].

[35] A. Sen, Stable non-BPS states in string theory, JHEP 06 (1998) 007 [hep-th/9803194] [INSPIRE].

[36] A. Sen, Stable non-BPS bound states of BPS D-branes, JHEP 08 (1998) 010 [hep-th/9805019] [INSPIRE].

[37] A. Sen, $\mathrm{SO}(32)$ spinors of type-I and other solitons on brane-anti-brane pair, JHEP $0 \mathbf{9}$ (1998) 023 [hep-th/9808141] [INSPIRE].

[38] M. Hindmarsh and T.W.B. Kibble, Beads on strings, Phys. Rev. Lett. 55 (1985) 2398 [INSPIRE].

[39] D. Tong, Monopoles in the Higgs phase, Phys. Rev. D 69 (2004) 065003 [hep-th/0307302] [INSPIRE]. 
[40] M. Cipriani and T. Fujimori, Effective action of non-Abelian monopole-vortex complex, arXiv: 1207.2070 [INSPIRE].

[41] J. Evslin, K. Konishi, M. Nitta, K. Ohashi and W. Vinci, Non-Abelian vortices with an Aharonov-Bohm effect, JHEP 01 (2014) 086 [arXiv:1310.1224] [INSPIRE].

[42] Y. Isozumi, M. Nitta, K. Ohashi and N. Sakai, All exact solutions of a 1/4 Bogomol'nyi-Prasad-Sommerfield equation, Phys. Rev. D 71 (2005) 065018 [hep-th/0405129] [INSPIRE].

[43] M. Eto, Y. Isozumi, M. Nitta, K. Ohashi and N. Sakai, Manifestly supersymmetric effective Lagrangians on BPS solitons, Phys. Rev. D 73 (2006) 125008 [hep-th/0602289] [INSPIRE].

[44] M. Eto, T. Fujimori, M. Nitta, K. Ohashi and N. Sakai, Higher derivative corrections to non-Abelian vortex effective theory, Prog. Theor. Phys. 128 (2012) 67 [arXiv:1204.0773] [INSPIRE].

[45] M. Nitta and W. Vinci, Non-Abelian monopoles in the Higgs phase, Nucl. Phys. B 848 (2011) 121 [arXiv: 1012.4057] [INSPIRE].

[46] E.R.C. Abraham and P.K. Townsend, Q kinks, Phys. Lett. B 291 (1992) 85 [inSPIRE].

[47] E.R.C. Abraham and P.K. Townsend, More on $Q$ kinks: a $(1+1)$-dimensional analog of dyons, Phys. Lett. B 295 (1992) 225 [InSPIRE].

[48] J.K. Perring and T.H.R. Skyrme, A model unified field equation, Nucl. Phys. 31 (1962) 550 [INSPIRE].

[49] M. Eto, T. Fujimori, M. Nitta, K. Ohashi and N. Sakai, Domain walls with non-Abelian clouds, Phys. Rev. D 77 (2008) 125008 [arXiv:0802.3135] [INSPIRE].

[50] E. Radu and M.S. Volkov, Existence of stationary, non-radiating ring solitons in field theory: knots and vortons, Phys. Rept. 468 (2008) 101 [arXiv:0804.1357] [INSPIRE].

[51] M. Eto, Y. Isozumi, M. Nitta, K. Ohashi and N. Sakai, Instantons in the Higgs phase, Phys. Rev. D 72 (2005) 025011 [hep-th/0412048] [INSPIRE].

[52] S. Kim, K.-M. Lee and H.-U. Yee, Supertubes in field theories, Phys. Rev. D 75 (2007) 125011 [hep-th/0603179] [INSPIRE].

[53] M. Eto, Y. Isozumi, M. Nitta and K. Ohashi, 1/2, 1/4 and 1/8 BPS equations in SUSY Yang-Mills Higgs systems: field theoretical brane configurations, Nucl. Phys. B 752 (2006) 140 [hep-th/0506257] [INSPIRE].

[54] N.H. Christ, A.H. Guth and E.J. Weinberg, Canonical formalism for gauge theories with application to monopole solutions, Nucl. Phys. B 114 (1976) 61 [INSPIRE]. 